九州大学学術情報リポジトリ

Kyushu University Institutional Repository

\title{
Sampling Distributions of Statistics Associated with a Fractile Graphic Method
}

Kitagawa, Toshio

Kyushu University

https://doi.org/10.5109/12989

出版情報: 統計数理研究. 9 (1)，pp. 10-42，1960-03. Research Association of Statistical Sciences バージョン:

権利関係 : 


\title{
SAMPLING DISTRIBUTIONS OF STATISTICS ASSOCIATED WITH A FRACTILE GRAPHIC METHOD
}

By

\author{
Tosio KITAgaWa
}

(Received December 20, 1959)

\section{Introduction}

0.1. Fundamental notions in fractile graphic analysis. In one of his lecture notes given in Japan 1958, November, P. C. Mahalanobis [1] stated his idea on fractile graphical analysis, where the notions of fractile group, fractile graphs, error area and separation play the fundamental rôles. Since these notes have not been published and their circulation seems to be limited, we shall beginn with a quotation of the original enunciations by $P$. C. Mahalanobis [1], 2.1 5.1.

(a) Fractile groups. " Suppose each sub-sample consists of $\boldsymbol{n}$ elementary units, each unit being a pair of values of the two variates $x$ and $y$. Consider the first sub-sample of, say, $n$ sample-units. Rank them in order of ascending values of $x$. It is now possible to divide the $n$ units into $g$ groups $(1,2, \ldots, i, \ldots, g)$, each of equal number, say $n^{\prime}$; so that $n=g n^{\prime}$. These may be called fractile groups." (MAHALANoBIs [1], 2.1 2.2)

(b) Fractile graphs, $\boldsymbol{G}(1)$ and $\boldsymbol{G}(2)$. "Next calculate the mean value (or median) of $n^{\prime}$ values of $y$ in each group to give in the first subsample the values $y_{1}^{\prime}, y_{2}^{\prime}, \ldots, y_{g}^{\prime}$ corresponding to the serial number of the groups $1,2, \ldots, i, \ldots, g$. Take $g$ equi-distant points $(1,2, \ldots, i, \ldots, g)$ on the $x$-axis to represent the $g$ groups; and plot the corresponding values of $y_{1}^{\prime}, y_{2}^{\prime}, \ldots, y_{g}^{\prime}$. Finally, draw straight lines to join each pair of adjoining points $y_{1}^{\prime}$ and $y_{2}^{\prime} ; y_{2}^{\prime}$ and $y_{3}^{\prime} ; \ldots, y_{i-1}^{\prime}$ and $y_{i}^{\prime} ; \ldots, y_{g-1}^{\prime}$ and $y_{g}^{\prime}$. This connected chain of lines will be called the fractile graph $G(1)$. Now consider the second sub-sample. It is possible to go through a similar process of ranking the sample units in order of ascending values of $x$; dividing them after ranking into $g$ groups each of equal number $\boldsymbol{n}^{\prime}$; calculating the values of $\boldsymbol{y}_{1}^{\prime \prime}$, $y_{2}^{\prime \prime}, \ldots, y_{g}^{\prime \prime}$; and drawing a second fractile graph $G(2)$. We can then have two sub-sample graphs $G(1)$ and $G(2)$, which have equal statistical validity." (MAHALANOBIS [1], 3. $\sim 3.3$ )

(c) Fractile graph $G(1,2)$. “It is also possible to mix together the 
two sub-samples to form a combined sample, and rank the sample units again; divide into $g$ groups of equal number $\left(2 n^{\prime}\right)$; calculate the new values of $y_{1}, y_{2}, \ldots, y_{g}$; and draw the fractile graph $G(1,2)$ for the two sub-samples taken together, that is, for the combined sample." (MAHALANOBIS [1], 3.4.).

(d) Error areas. "It is possible to measure on paper the area bounded by the two sub-sample fractile graphs $G(1)$ and $G(2)$. We shall (semi-intuitively) call this area $a(1,2)$ as the "error" to be associated with the combined fractile graph $G(1,2)$." (MAHALANOBIs [1], 4.1).

(e) Separation. "It is further possible to consider a second population from which a pair of interpenetrating sub-samples are drawn, and a second set of fractile graphs, say $G^{\prime}(1), G^{\prime}(2)$ and $G^{\prime}(1,2)$ are constructed in exactly the same way. The area bounded by $G^{\prime}(1)$ and $G^{\prime}(2)$ would give the second error area $a^{\prime}(1,2)$ to be associated with the second pooled graph $G^{\prime}(1,2) . "$ (MAHALANOBIS [1], 5.1 5.2)

0.2. The needs of sampling theories for fractile graphic analysis. The fractile graph analysis has a lot of practical applications to statistical analysis of data, as has been shown by various examples of P. C. MAHALANoBis [1] [2].

"It is also rich of flexibilities because of minor restrictions on the mutual relation between $x$ and $y$." (MAHALANOBIs [1], 7.1 7.3) Before claiming a powful or statistical tool as a substitute or even an improved alternative for current statistical analysis, it is, however, required to establish some exact observations on sampling distributions of statistics associated with the notions $(a) \sim(e)$. Otherwise, testings of hypothesis and estimation theorems could not be developed in the standard of the current inference theories. There remain theorefore several fundamental observations still unestabished.

0.3. The Surmises by Mahalanobis. P. C. Mahalanobis [1] gave a number of semi-intuitive surmises some of which are experimentally studied. Among others he gave the following ones:

$\left(1^{\circ}\right)$ "The area $a(1,2)$ which has been called the error associated with $G(1,2)$ would decrease statistically in proportion to $n^{\prime-1 / 2}$ with increasing size of the sample $n^{\prime}$ of each group (when $g$ is constant) ; and would increase in proportion to $g$, (when $\boldsymbol{n}^{\prime}$ is kept constant) ; as a first approximation." (Mahalanobis [1], 6.2)

$\left(2^{\circ}\right)$ "The combined fractile graph $G(1,2)$ would tend statistically to lie more and more within the area $a(1,2)$ with increasing values of $n^{\prime}$ (with $g$ constant)." (MAHALANOBIs [1], 6.3)

$\left(3^{\circ}\right)$ "The number of points of intersection of $G(1)$ and $G(2)$ would tend statistically to be distributed like changes in " runs" of heads and tails in $g$ throws of an unbiassed coin. " (MAHALANOBIs [1], 6.4)

$\left(4^{\circ}\right)$ "The error to be associated with the "Separation", $S(1,2)$, to 
be called, say, $E$ can be found in the usual way from the two error areas, $a(1,2)$ and $a^{\prime}(1,2)$, associated respectively with the two combined fractile graphs, $G(1,2)$ and $G^{\prime}(1,2)$, representing respectively the two populations from which the two pairs of sub-samples are drawn. That is, it is possible to take $E=\sqrt{\left[a^{2}(1,2)+a^{\prime 2}(1,2)\right]}$. " (Mahalanobis [1], 6.5)

$\left(5^{\circ}\right)$ " To test the significance of the observed separation, it is possible to use the criterion $S^{2} / E^{2}$ which would tend to be distributed, as a first approximation, like Chi-square." (MAHALANOBIS [1], 6.6)

0.4. Summary of the present paper. This paper concerns with the more fundamental aspects of sampling distributions associated with statistics of fractile graphic analysis rather than with the Mahalanobis surmises. Let us start with a random sample $\left\{\left(x_{i}, y_{i}\right)\right\}(i=1,2, \ldots, n)$ of size $n$ from a bivariate population. Let $\left\{x_{(i)}\right\}$ be the order statistics defined by $\left\{x_{i}\right\}$ (i $=1,2, \ldots, n)$ such that $x_{(1)} \leqq x_{(2)} \leqq \ldots \leqq x_{(n)}$ which is a rearrangement of $n$ values $x_{1}, x_{2}, \ldots, x_{n}$ in the ascending order. Each $y_{j}$ associated with $x_{j}$ in our random sample will be denoted by $y_{(i)}$ when $x_{j}$ is corresponding to $x_{(i)}$ in the rearrangement. Let us divide the set $\left\{y_{(j)}\right\} \quad(j=1,2, \ldots, n)$ into $g$ subsets $G_{i}(i=1,2, \ldots, g)$ where each $G_{i}$ consists of $\left\{y_{(N i-1+k)}\right\} \quad(k=1,2, \ldots$, $\left.n_{i}^{\prime}\right)$, such that $N_{0}=0, N_{i-1}=n_{1}^{\prime}+n_{2}^{\prime}+\ldots+n_{i-1}^{\prime}(i=1,2, \ldots g)$ with $n_{1}^{\prime}+n_{2}^{\prime}+\ldots$ $+n_{g}^{\prime}=n$, and let us define

$$
y_{(i)}=\left(y_{\left(N_{i-1}+1\right)}+y_{\left(N_{i-1}+2\right)}+\ldots+y_{\left(N_{i}\right)}\right) / n_{i}^{\prime},(i=1,2, \ldots, g) .
$$

We are mainly (not exclusively) concerned with the case when $n_{1}^{\prime}=n_{2}^{\prime}$ $\ldots=\boldsymbol{n}_{g}^{\prime}=n^{\prime}$ and $g n^{\prime}=n$.)

Let us consider the case when $n_{1}^{\prime}=n_{2}^{\prime}=\ldots=n_{g}^{\prime}=n$ and $g n^{\prime}=n$. In virtue of the statistics $\left\{\boldsymbol{y}_{(i)}\right\}$ we can define the area $S$ which corresponds to the error area in the terminology of MAHAlanobis [1]. The area $S$ is the sum of $(g-1)$ areas $\left\{S_{k}\right\}(k=1,2, \ldots, g-1)$ where each $S_{k}$ is defined with respect to the four points (1.11). This definition can be generalised to the separation in the sense of MAHALANOBIS, as we will show in $\S 1$.

The integrals (3.21) and (3.22) are directly associated with the evaluations of $E\left\{S_{k}^{\prime}\right\}$ under our particular situations appealing to the asymptotic normality.

The results in $\$ 1$ are concerned with the exact representations of $\left(1^{\circ}\right)$ the simultaneous distribution functions of some of the statistics $\left\{y_{(i)}\right\},\left(2^{\circ}\right)$ the distribution function of $\left(\boldsymbol{y}_{(h+1)}+\ldots+\boldsymbol{y}_{(k))}\right) /\left(k_{c}-h\right), \quad\left(3^{\circ}\right) E\left\{S_{k}^{l}\right\}, \quad\left(4^{\circ}\right) E$ $\left\{S_{k 1}^{u 1} S_{k 2}^{22}\right\},\left(5^{\circ}\right) E\left\{S_{k 1}^{u 1} S_{k 2}^{i 2} S_{k 3}^{z 3}\right\},\left(6^{\circ}\right) E\left\{S_{k 1}^{u} S_{k 2}^{i 2} S_{k 3}^{i 3} S_{k 4}^{44}\right\}$, where $l_{i} \geqq 1$ and $1 \leqq k_{1}$ $<k_{2}<k_{3}<k_{4} \leqq g$, and hence $\left(7^{\circ}\right) E\left\{S^{\prime}\right\} \quad(l=1,2,3,4)$.

In $\S 2$ the asymptotic form of the multivariate probability density function of $\left(\bar{y}_{\left.\left(k_{1}\right\rangle\right)}, \bar{y}_{\left(k_{2}\right)}, \ldots, y_{\left(k k_{1}\right)}\right)$ is discussed on the basis of the Assumptions I and II. The Assumption I refers to the expression of the simultaneous probability density functions in the form of (1.32), and will yield us normal 
approximations to the multivariate probability density functions. The Assumption II is introduced so as to be able for us to appeal to the well-known Theorem of MOSTELLER [1] concerning the limiting simulataneous distribution of order statistics. Here the asymptotic normality is established in Theorem 2.1 for the simultaneous probability distribution of the statistics $\left(\bar{y}_{\left(k k_{1}\right)}, y_{\left(k k_{2}\right)}, \ldots, \bar{y}_{(k k h)}\right)$ as $n^{\prime}$ tends to infinity. In view of the results given in Theorem 2.1 and in $\S 1.8$, the evaluations of the integrals enunciated in the right-hand sides of $\left(1^{\circ}\right) \sim\left(15^{\circ}\right)$ in $\S 1.8$ such as those given in $(1.521) \sim(1.524)$ are reduced to those associated with the multivariate normal populations, provided that we are interested with a fairly large or moderately large $n^{\prime}$. In $\$ 3.1$ we start with a general procedure to evaluate the integrals of the type (3.01) associated with an $n$-dimentional multivariate normal distribution. In $\S 3.2$ the procedure is applied to the two-dimensional case, that is, $n=2$.

The results given in $\$ 3.2$ can directly serve to give asymptotic evaluations of the first two moments of the area $S$, that is, $E\left\{S^{l}\right\} \quad(l=1,2)$ under the assumption of asymptotic normality. The general procedure of evaluating the integral of the type (3.01) can also serve to calculate the asymptotic values of the third and the fourth moments $E\left\{S^{l}\right\} \quad(l=3,4)$. In view of tremendous numbers of various types of the integrals in the right-hand sides of (1.47) and (1.48), however, the problem is remaining unsolved in this paper how to evaluate the sums of them, although their summands can be shown to have certain rather complicated asymptotic values.

In the consequence the surmises by MAHALANOBIs [1] are not claimed to have been solved in any definite way in this paper, but it is hoped that our formulation and allied analysis will serve to make an approach to them. Numerical aspects are shown in $\S 4$ to give certain constants such as variances, covariances and correlation coefficients associated with the multivariate normal distribution derived from a graphical fractile analysis when the sample size is 20 .

It is also to be noted that $\mathrm{K}$. TAKEUCHI [1] discussed the surmises by MAHALANOBIS on the basis of regression model which is different from the formulation of the present paper, but had reached the results which are essentially the same with ours given in $(3.461) \sim(3.462)$.

\section{$\S 1$. The statistics $\left\{y_{(i) ~}\right\}$ and $\left\{S_{k}\right\}$ defined for a bivariate distribu- tion $(X, Y)$ with respect to the order statistics of $X$.}

1.1. Let $\left\{\left(x_{i}, y_{i}\right)\right\} \quad(i=1,2, \ldots, n)$ be a random sample of size $n$ from a bivariate population. Let $\left\{x_{(i)}\right\}$ be the order statistics defined by $\left\{x_{i}\right\}$ ( $i$ $=1,2, \ldots, n)$ such that $x_{(1)} \leqq x_{(2)} \leqq \ldots \leqq x_{(n)}$ which is a rearrangement of $n$ values $x_{1}, x_{2}, \ldots, x_{n}$ in the ascending order. Each $y_{j}$ associated with $x_{j}$ in our random sample will be denoted by $y_{(i)}$ if $x_{j}$ is corresponding to $x_{(i)}$ in the rearrangement. Consequently we shall have the rearrangement of our 
sample :

$$
\left(x_{(1)}, y_{(1))}\right),\left(x_{(2)}, y_{(2)}\right), \ldots,\left(x_{(n)}, y_{(n))}\right) .
$$

According to Mahalanobis [1], we may devide the set $\left\{\boldsymbol{y}_{(j)}\right\}(j=1,2, \ldots$, $n$ ) into $g$ subsets $G_{i}(i=1,2, \ldots, g)$ where each $G_{i}$ consists of $\left\{y_{((N i-1+k))}\right\}$ $\left(k=1,2, \ldots, n_{i}^{\prime}\right)$, such that $N_{0}=0, N_{i-1}=n_{1}^{\prime}+n_{2}^{\prime}+\ldots+n_{i-1}^{\prime}(i=1,2, \ldots, g)$ with $\boldsymbol{n}_{1}^{\prime}+\boldsymbol{n}_{2}^{\prime}+\ldots+\boldsymbol{n}_{g}^{\prime}=\boldsymbol{n}$, and let us define

$$
\bar{y}_{(i)\rangle}=\left(y_{\left(\left(N_{i-1}+1\right)\right)}+y_{\left(\left\langle N_{i-1}+2\right)\right.}+\quad+y_{\left(N_{i}\right)}\right) / n_{i}^{\prime} .(i=1,2, \ldots, g)
$$

In what follows simply for the sake of simplicity we are mainly (not exclusively) concerned with the case when $\boldsymbol{n}_{1}^{\prime}=\boldsymbol{n}_{2}^{\prime}=\ldots=\boldsymbol{n}_{g}^{\prime}=\boldsymbol{n}^{\prime}$ and $\boldsymbol{g} \boldsymbol{n}^{\prime}=\boldsymbol{n}$.

1.2. Let the probability density function of our bivariate population be denoted by $f(x, y)$, and let us denote the marginal probability distribution density of $X$ by

$$
f_{1}(x)=\int_{-\infty}^{\infty} f(x, y) d y
$$

and the conditional probability density by $f_{1}(y \mid x)$ by

$$
f_{1}(y \mid x)=f(x, y) / f_{1}(x) .
$$

The probability distribution of $X$ is then given by

$$
F_{1}(x)=\int_{-\infty}^{x} f_{1}(x) d x=\int_{-\infty}^{x} d x \int_{-\infty}^{\infty} f(x, y) d y .
$$

We have consequently

$$
\begin{aligned}
& \operatorname{Pr} .\left\{y<y_{(k))}<y+d y, x<x_{(k)}<x+d x\right\} \\
& =\frac{n !}{(k-1) !(n-k) !}\left(F_{1}(x)\right)^{k-1} f(x, y)\left(1-F_{1}(x)\right)^{n-k} d x d y \\
& \operatorname{Pr} .\left\{y<y_{(k))}<y+d y\right\} \\
& =\frac{n !}{(k-1) !(n-k) !}\left(\int_{-\infty}^{\infty}\left(F_{1}(x)\right)^{k-1} f(x, y)\left(1-F_{1}(x)\right)^{n-k} d x\right) d y
\end{aligned}
$$

and hence, for $l \geqq 1$,

$$
\begin{aligned}
& E\left\{y_{(k))}^{l}\right\} \\
& =\int_{-\infty}^{\infty} y^{l} d y \frac{n !}{(k-1) !(n-k) !} \int_{-\infty}^{\infty}\left(F_{1}(x)\right)^{k-1} f(x, y)\left(1-F_{1}(x)\right)^{n-k} d x,
\end{aligned}
$$

provided that the mements are assumed to exist.

1.3. The simultaneous probability density density function of two statistics $y_{(i))}$ and $y_{(j))}$ is now given by

(1.09) $\quad g\left(y_{i}, y_{j}\right) d y_{i} d y_{j}$

$$
\equiv \operatorname{Pr} .\left\{y_{i}<y_{(i))}<y_{i}+d y_{i}, y_{j}<y_{(j)}<y_{j}+d y_{j}\right\}
$$




$$
\begin{aligned}
& =\frac{n !}{(i-1) !(j-i-1) !(n-j) !} \int_{-\infty}^{\infty} d x_{1} \int_{x_{1}}^{\infty}\left(F\left(x_{1}\right)\right)^{i-1}\left(F\left(x_{2}\right)-F\left(x_{1}\right)\right)^{j-i-1} \\
& \left(1-F\left(x_{2}\right)\right)^{n-j} f\left(x_{1}, y_{i}\right) f\left(x_{2}, y_{j}\right) d x
\end{aligned}
$$

for $i<j$.

A product moment of $y_{(i)}$ and $y_{(j))}$ is now given by

$$
E\left\{y_{(i)}^{p} y_{(j)}^{q}\right\}=\int_{-\infty}^{\infty} \int_{-\infty}^{\infty} y_{i}^{p} y_{j}^{q} g\left(y_{i}, y_{j}\right) d y_{i} d y_{j},
$$

provided that it is assumed to exist.

1.4. Now let us consider two random samples of the same size $\boldsymbol{n}$ from a bivariate population, which we denote by $\left\{\left(x_{i}, y_{i}\right)\right\}(i=1,2, \ldots, n)$ and $\left\{\left(x_{i}^{\prime}, z_{i}\right)\right\}(i=1,2, \ldots, n)$ respectively. According to the statistical procedure defined in $\S 1.1$, we can define $\left(\bar{y}_{(1)}, y_{(2)}, \ldots, \bar{y}_{(g)}\right)$ and $\left(\bar{z}_{(1)}, z_{(2)}, \ldots, \bar{z}_{(g)}\right)$.

We are interested with the area $S$ defined in the following way according to Mahalanobis [1]. points :

First of all let the $k$ th subarea $S_{k}$ be defined with respect to the four

$$
\left(k d, \bar{y}_{(k k))}\right),\left((k+1) d, y_{(k+1))}\right),\left(k d, \bar{z}_{(k k)}\right),\left((k+1) d, \bar{z}_{(k+1)}\right),
$$

and then let us define $S$ by

$$
S=S_{1}+S_{2}+\ldots+S_{g-1} \text {. }
$$

Now let each $S_{k}$ be defined in the following way. There are two cases to be distinguished with each other. Let us consider two straight lines

$$
\begin{aligned}
& y-y_{(k k)}=\frac{\bar{y}_{(k+1)}-\bar{y}_{(k))}}{d}(x-k d) \\
& y-\bar{z}_{(k)}=\frac{\bar{z}_{(k+1)}-\bar{z}_{(k k))}}{d}(x-k d) .
\end{aligned}
$$

Let the abscissa of the point where the two straight lines meet with each other be denoted by $x_{k, k+1}$. For our purpose the case $\left(1^{\circ}\right): x_{k, k+1}<k d$ or $x_{k, k+1}>(k+1) d$, and the case $\left(2^{\circ}\right): k d<x_{k, k+1}<(k+1) d$, must be distinguished with each other.

The case $\left(1^{\circ}\right)$ : This case will happen under the following two mutually exclusive cases

$$
\begin{cases}A_{1}: & \bar{y}_{(k i)} \geqq \bar{z}_{(k)} \bar{y}_{(k+1)} \geqq \bar{z}_{(k+1)} \\ A_{2}: & \bar{y}_{(k \lambda)}<\bar{z}_{(k k)} \bar{y}_{(k k+1)}<\bar{z}_{(k+1))},\end{cases}
$$

and $S_{k}$ is defined by

$$
S_{k}=\boldsymbol{d}\left(\left|\bar{y}_{(k)}-\bar{z}_{(k k)}\right|+\left|\bar{y}_{(k+1)}-\bar{z}_{\langle k+1)}\right|\right) / 2
$$

The case $\left(2^{\circ}\right)$ : This case will happen under the following two mutually exclusive cases: 


$$
\left\{\begin{array}{lll}
B_{1}: & \bar{y}_{(k \lambda)} \geq z_{(k \lambda)}, & \bar{y}_{(k+1)}<\bar{z}_{(k+1)} \\
B_{2}: & \bar{y}_{\langle k\rangle}<\bar{z}_{(k k)}, & y_{(k+1)}>z_{(k+1)},
\end{array}\right.
$$

and $S_{k}$ is given by

$$
S_{k}=\frac{d}{2} \cdot \frac{\bar{y}_{(k i)}-\bar{z}_{(k k)}{ }^{2}+\bar{y}_{(k+1)}-\left.\bar{z}_{(k+1)}\right|^{2}}{y_{(k k)}-\bar{z}_{(k k))}+\left|y_{(k+1)}-z_{(k+1) !}\right|} \cdot
$$

Let the simultaneous probability density function of $\bar{y}_{(k))}$ and $\bar{y}_{(k+1)}$ be denoted by $p_{k}(u, v)$, that is,

$$
P\left\{u<\bar{y}_{(k)}<u+d u, v<\bar{y}_{(k+1)}<v+d v\right\} \equiv p_{k}(u, v) d u d v .
$$

Let us define

$$
\begin{aligned}
E\{ & \left.S_{k}^{l}\right\} \equiv \int_{-\infty}^{\infty} \int_{-\infty}^{\infty} S_{k} p_{k}\left(\bar{y}_{k}, y_{k+1}\right) p_{k}\left(\bar{z}_{k}, z_{k+1}\right) d \bar{y}_{k} d \bar{z}_{k} d \bar{y}_{k+1} d \bar{z}_{k+1} \\
= & \left(\frac{d}{2}\right)^{l} \int_{D} \int_{\left(A_{1}\right)}((u-U)+(v-V))^{l} p_{k}(u, v) p_{k}(U, V) d u d v d U d V \\
& +\left(\frac{d}{2}\right)^{l} \int_{D} \int_{\left(A_{2}\right)}\left((U-u)+(V-v)^{l} p_{k}(u, v) P_{k}(U, V) d u d v d U d V\right. \\
& +\left(\frac{d}{2}\right)^{l} \int_{D} \int_{\left(B_{1}\right)}\left(\frac{(u-U)^{2}+(v-V)^{2}}{(u-U)+(V-v)}\right)^{l} p_{k}(u, v) p_{k}(U, V) d u d v d U d V \\
& +\left(\frac{d}{2}\right)^{l} \int_{D} \int_{\left(B_{2}\right)}\left(\frac{(u-U)^{2}+(v-V)^{2}}{(U-u)+(v-V)}\right)^{l} p_{k}(u, v) p_{k}(U, V) d u d v d U d V \\
\equiv & I^{l}\left(A_{1}\right)+I_{l}\left(A_{2}\right)+I_{l}\left(B_{1}\right)+I_{l}\left(B_{2}\right), \text { say },
\end{aligned}
$$

provided it does exist, where the domains $\mathfrak{D}\left(A_{i}\right)$ and $\mathfrak{D}\left(B_{i}\right)(i=1,2)$ are defined as follows:

Let us put

$$
p_{k}^{(1,1)}(u, v) \equiv \int_{-\infty}^{\infty} \int_{-\infty}^{\infty} p_{k}(x+u, y+v) p_{k}(x, y) d x d y .
$$

Then we have, in view of (1.19),

$$
\begin{aligned}
& I_{l}\left(A_{1}\right)=\left(\frac{d}{2}\right)^{l} \int_{0}^{\infty} \int_{0}^{\infty}(u+v)^{l} p_{k}^{(1,1)}(u, v) d u d v \\
& I_{l}\left(A_{2}\right)=\left(\frac{d}{2}\right)^{l} \int_{0}^{\infty} \int_{0}^{\infty}(u+v)^{l} p_{k}^{(1,1)}(-u,-v) d u d v \\
& I_{l}\left(B_{1}\right)=\left(\frac{d}{2}\right)^{l} \int_{0}^{\infty} \int_{0}^{\infty}\left(\frac{u^{2}+v^{2}}{u+v}\right)^{l} p_{k}^{(1,1)}(u,-v) d u d v
\end{aligned}
$$




$$
I_{l}\left(B_{2}\right)=\left(\frac{d}{2}\right)^{l} \int_{0}^{\infty} \int_{0}^{\infty}\left(\frac{u^{2}+v^{2}}{u+v}\right) p_{k}^{(1,1)}(-u, v) d u d v .
$$

1.5. Let us now calculate the probability density funotion of $\bar{y}_{(k)\rangle}$. Let us denote by $E_{(h, k)}^{x}$ and $E_{(h k)}^{y}$ the events defined by

$$
\begin{aligned}
E_{(h, k)}^{x}: & t_{1}<x_{(h+1)}<t_{1}+d t_{1}, t_{2}<x_{(h+2)}<t_{2}+d t_{2}, \ldots, \\
& t_{k-h}<x_{(k)}<t_{k-h}+d t_{k-h} \\
E_{(h, k)}^{y}: & y_{1}<y_{(h+1))}<y_{1}+d y_{1}, y_{2}<y_{(h+2))}<y_{2}+d y_{2}, \ldots, \\
& y_{k-h}<y_{(k))}<y_{k-h}+d y_{k-h}
\end{aligned}
$$

respectively. The probability density element for the simultaneous occurrence of $E_{(n, k)}^{x}$ and $E_{(n, k)}^{y}$ is given by

$$
\operatorname{Pr} .\left\{E_{(n, k)}^{x} \cap E_{(h, k)}^{\prime \prime}\right\}
$$

$$
\begin{aligned}
& =\frac{n !}{h !(n-k) !}\left(F_{1}\left(t_{1}\right)\right)^{n}\left(1-F\left(t_{k-h}\right)\right)^{n-k} \\
& \cdot f\left(t_{1}, y_{1}\right) f\left(t_{2}, y_{2}\right), \ldots, f\left(t_{k-h}, y_{k-h}\right) \prod_{i=1}^{k-n} d t_{i} d y_{i} .
\end{aligned}
$$

Consequently we have

$$
\begin{aligned}
\operatorname{Pr} . & \left\{y<y_{(n+1)}+y_{(n+2)}+\ldots+y_{(k))}<y+d y, E_{n k}^{(x)}\right\} \\
& =\frac{n !}{n !(n-k) !}\left(F_{1}\left(t_{1}\right)\right)^{n}\left(1-F_{1}\left(t_{k-h}\right)\right)^{n-k} \prod_{i=h+1}^{k} f_{1}\left(t_{i}\right) d t_{i} \\
& \iint_{y<y_{1}+y_{2}+\cdots+y_{k-h}<y+d y} \ldots \int_{f}\left(y_{1} t_{1}\right) f\left(y_{2} \mid t_{2}\right) \ldots f\left(y_{k-n} \mid t_{k-h}\right) d y_{1} d y_{2} \ldots d y_{k-h},
\end{aligned}
$$

where the integral in the righthand side can be written by convolution

$$
f_{t_{1}} * f_{t_{2}}^{*} \ldots * f_{t_{k-h}}(y) d y,
$$

where $f_{t i} \equiv f\left(y \mid t_{i}\right)$.

Fininally we have

$$
\begin{aligned}
& \operatorname{Pr}_{\cdot}\left\{y<\frac{y_{(h+1)}+y_{(h+2)}+\ldots+y_{(k k))}}{k-h}<y+d y\right\} \\
= & \frac{n !(k-h)}{h !(k-h) !(\boldsymbol{n}-k) !} \\
& \cdot \iint_{-\infty<t_{1}<t_{2}<\cdots t k-h<\infty} \ldots \int_{1}\left(F_{1}\left(t_{1}\right)\right)^{h}\left(1-F_{1}\left(t_{k-h}\right)\right)^{n-k} \\
& f_{t_{1}}^{*} f_{t_{2}}{ }^{*} \ldots f_{t_{k-h}}((k-h) y) d t_{1} \ldots d t_{k-h}
\end{aligned}
$$

For $h=(i-1) n^{\prime}, k=i n^{\prime}$, (1.30) gives us the probability distribution element $\bar{y}_{(i)}$, that is,

$$
\operatorname{Pr} .\left\{\boldsymbol{y}<\bar{y}_{(k))}<u+d u\right\} \equiv h_{k}(u) d u
$$




$$
\begin{aligned}
& =\frac{n ! n^{\prime} d u}{\left((k-1) n^{\prime}\right) ! n^{\prime} !\left((g-\bar{k}) n^{\prime}\right) !} \\
& \left.\iint_{-\infty<t_{1}<t_{2}<\cdots<t_{n^{\prime}<\infty}} \ldots \int_{f_{t_{1}}{ }^{*} f_{t_{2}}{ }^{*} \ldots{ }^{*}{ }_{t n^{\prime}}\left(n^{\prime} u\right) d t_{1} \ldots d t_{n^{\prime}}}\left(t_{1}\right)\right)^{(k-1) n^{\prime}}\left(1-F_{1}\left(t_{n^{\prime}}\right)\right)^{(y-k) n^{\prime}} \prod_{i=1}^{n^{\prime}} f_{1}\left(t_{i}\right)
\end{aligned}
$$

Similarly we have

$$
\begin{gathered}
\operatorname{Pr} .\left\{u<\bar{y}_{(k k))}<u+d u, v<\bar{y}_{(k+1))}<v+d u\right\} \equiv p_{k}(u, v) d u d v \\
=\frac{n ! n^{\prime} d u n^{\prime} d v}{\left((k-1) n^{\prime}\right) ! n^{\prime} ! n^{\prime} !((g-k-1)} \overline{\left.n^{\prime}\right) !} \\
\iint_{-\infty<t_{1}<t_{2}<\cdots<t_{2 i}{ }^{\prime}<\infty} \ldots \int_{t^{\prime}}\left(F_{1}\left(t_{1}\right)\right)^{(k-1) n^{\prime}}\left(1-F_{1}\left(t_{2 n^{\prime}}\right)\right)^{(g-k-1) n^{\prime}} \prod_{i=1}^{2 n^{\prime}} f_{1}\left(t_{i}\right) \\
f_{t_{1}}^{*} f_{t_{2}}^{*} \ldots f_{t n^{\prime}}\left(n^{\prime} u\right) \\
f_{t_{n^{\prime}+1}} * f_{t_{n^{\prime}+2}}^{*} \ldots f_{t_{n^{\prime}}}\left(n^{\prime} v\right) d t_{1} d t_{2} d t_{2 n^{\prime}} .
\end{gathered}
$$

1.6. We shall now enter into the discussion of the separation in the sense of MAHALANOBIS [1]. Let us consider two bivariate populations $\Pi_{1}$ and $\Pi_{2}$ from each of which let us draw independently a random sample of the same size $n$. Let us denote these two samples from $\Pi_{1}$ and $\Pi_{2}$ by $\left\{\left(x_{i}, y_{i}\right)\right\}$ $(i=1,2, \ldots, n)$ and $\left\{\left(x_{i}^{\prime}, z_{i}\right)\right\}(i=1,2, \ldots, n)$ respectively.

We can define the area $S$ just as in (1.12) for which the expressions (1.15) and (1.17) hold true under their respective conditions. Now let us define

$$
\begin{aligned}
& \operatorname{Pr} .\left\{y<\bar{y}_{(k))}<y+d y\right\} \equiv h_{1, k}(y) d y \\
& \operatorname{Pr} .\left\{z<\bar{z}_{(k k)}<z+d z\right\} \equiv h_{2, k}(z) d z \\
& \operatorname{Pr} .\left\{u<\bar{y}_{(k))}<u+d u, v<\bar{y}_{(k+1))}<v+d v\right\} \equiv p_{1, k}(u, v) d u d v \\
& \operatorname{Pr} .\left\{U<\bar{z}_{(k))}<U+d U, V<\bar{z}_{(k+1))}<V+d V\right\} \equiv p_{2, k}(U, V) d U d V,
\end{aligned}
$$

in order to distinguish the (possible) difference of the two populations $\amalg_{1}$ and $\mathrm{I}_{2}$.

Let us put

$$
p_{k}^{(12)}(u, v) \equiv \int_{-\infty}^{\infty} \int_{-\infty}^{\infty} p_{1, k}(x+u, y+v) p_{2, k}(x, y) d x d y .
$$

Then the argument sililar to that devoloped in $\$ 1.5$ gives us

$$
E\left\{S_{k}^{l}\right\}=I_{l}^{(1,2)}\left(A_{1}\right)+I_{l}^{(1,2)}\left(A_{2}\right)+I_{l}^{(1,2)}\left(B_{1}\right)+I_{l}^{(1,2)}\left(B_{2}\right) \text {, }
$$

where

$$
I_{l}^{(1,2)}\left(A_{1}\right)=\left(\frac{d}{2}\right)^{l} \int_{0}^{\infty} \int_{0}^{\infty}(u+v)^{l} p_{k}^{(1,2)}(u, v) d u d v
$$




$$
\begin{aligned}
& I_{l}^{(1,2)}\left(A_{2}\right)=\left(\frac{d}{2}\right)^{l} \int_{0}^{\infty} \int_{0}^{\infty}(u+v)^{l} p_{k}^{(1,2)}(-u,-v) d u d v \\
& I_{i}^{(1,2)}\left(B_{1}\right)=\left(\frac{d}{2}\right)^{l} \int_{0}^{\infty} \int_{0}^{\infty}\left(\frac{u^{2}+v^{2}}{u+v}\right) p_{k}^{(1,2)}(u,-v) d u d v \\
& I_{l}^{(1,2)}\left(B_{2}\right)=\left(\frac{d}{2}\right)^{l} \int_{0}^{\infty} \int_{0}^{\infty}\left(\frac{u^{2}+v^{2}}{u+v}\right) p_{k}^{(1,2)}(-u, v) d u d v
\end{aligned}
$$

provided that these integrals are assumed to exist.

1. 7. Let us consider two populations $\Pi_{1}$ and $\Pi_{2}$ introduced in $\$ 1.6$. The purpose of this paragraph is to decompose the first four moments of the statistic $S$ defined in (1.12) into the sums of some fundamental integrals. For the sake of convenience let us introduce the notations:

$$
\begin{aligned}
& E\left\{S_{k}^{L}\right\} \equiv\left(k^{l}\right) \\
& E\left\{S_{k 1}^{l_{1}} S_{k 2}^{L_{2}}\right\} \equiv\left(k_{1}^{l_{1}} k_{2}^{l_{2}}\right) \\
& E\left\{S_{k_{1}}^{l_{1}} S_{k 2}^{l_{2}} S_{k 3}^{l_{3}}\right\} \equiv\left(k_{1}^{l_{1}} k_{2}^{l_{3}} k_{3}^{l_{3}}\right) \\
& E\left\{S_{k_{1}}^{l_{1}} S_{k_{2}}^{l_{2}} S_{k_{3}}^{l_{3}} S_{k_{k}}^{l_{4}}\right\} \equiv\left(k_{1}^{l_{1}} k_{2}^{l_{2}} k_{3}^{l_{3}} k_{4}^{l_{4}}\right),
\end{aligned}
$$

where $l_{i} \geqq 1$ and $1 \leqq k_{1}<k_{2}<k_{3}<k_{4} \leqq g$.

In virtue of these notations we have

$$
\begin{aligned}
E\{S\} & =\sum_{k=1}^{g-1} E\left\{S_{k}\right\} \equiv \sum_{k=1}^{g-1}(k) \\
E\left\{S^{2}\right\} & =\sum_{k=1}^{g-1} E\left\{S_{k}^{2}\right\}+2 \sum_{k=1}^{g-2} E\left\{S_{k} S_{k+1}\right\}+2 \sum_{k=1}^{g-3} \sum_{j=k}^{g-1} E\left\{S_{k} S_{j}\right\} \\
& =\sum_{k=1}^{g-1}\left(k^{2}\right)+2 \sum_{k=1}^{g-2}(k(k+1))+2 \sum_{k=1}^{g-3} \sum_{j=k+2}^{g-1}(k j)
\end{aligned}
$$$$
E\left\{S^{3}\right\}=\sum_{k=1}^{g-1}\left(k^{3}\right)+3 \sum_{k=1}^{g-2}\left(k^{2}(k+1)\right)+3 \sum_{k=1}^{g-2}\left(k(k+1)^{2}\right)
$$$$
+3 \sum_{k=1}^{g-3} \sum_{j=k+2}^{g-1}\left(k^{2} j\right)+3 \sum_{k=1}^{g-3} \sum_{j=k+2}^{g-1}\left(k j^{2}\right)
$$$$
+6 \sum_{k=1}^{g-3}(k(k+1)(k+2))+6 \sum_{k=1}^{g-4} \sum_{j=k+3}^{g-1}(k(k+1) j)
$$$$
+6 \sum_{k=1}^{g-4} \sum_{j=k+2}^{g-2}(k j(j+1))+6 \sum_{k=1}^{g-5} \sum_{j=k+2}^{g-3} \sum_{h=j+2}^{g-1}(k j h)
$$

$$
\begin{gathered}
E\left\{S^{4}\right\}=\sum_{k=1}^{g-1}\left(k^{4}\right)+4 \sum_{k=1}^{g-2}\left(k^{3}(k+1)\right)+4 \sum_{k=1}^{g-2}\left(k(k+1)^{3}\right) \\
+12 \sum_{k=1}^{g-2}\left(k^{2}(k+1)^{2}\right)+4 \sum_{k=1}^{g-3} \sum_{j=k+2}^{g-1}\left(k^{3} j\right)
\end{gathered}
$$




$$
\begin{aligned}
& +4 \sum_{k=1}^{g-3} \sum_{j=k+2}^{g-1}\left(k j^{3}\right)+12 \sum_{k=1}^{g-3} \sum_{j=k+2}^{g-1}\left(k^{2} j^{2}\right) \\
& +12 \sum_{k=1}^{g-3}\left(k^{2}(k+1)(k+2)\right)+12 \sum_{k=1}^{g-3}\left(k(k+1)^{2}(k+2)\right) \\
& +12 \sum_{k=1}^{g-3}\left(k(k+1)(k+2)^{2}\right)+12 \sum_{k=1}^{g-4} \sum_{j=k+3}^{g-1}\left(k^{2}(k+1) j\right) \\
& +12 \sum_{k=1}^{g-4} \sum_{j=k+3}^{g-1}\left(k(k+1)^{2} j\right)+12 \sum_{k=1}^{g-4} \sum_{j=k+3}^{g-1}\left(k(k+1) j^{2}\right) \\
& +12 \sum_{k=1}^{g-4} \sum_{j=k+2}^{g-1}\left(k^{2} j(j+1)\right)+12 \sum_{k=1}^{g-4} \sum_{j=k+2}^{g-1}\left(k j^{2}(j+1)\right) \\
& +12 \sum_{k=1}^{g-4} \sum_{j=k+2}^{g-1}\left(k j(j+1)^{2}\right)+12 \sum_{k=1}^{g-5} \sum_{j=k+2}^{g-3} \sum_{h=j+2}^{g-1}\left(k^{2} j h\right) \\
& +12 \sum_{k=1}^{g-5} \sum_{j=k+2}^{g-3} \sum_{h=j+2}^{g-1}\left(k j^{2} h\right)+12 \sum_{k=1}^{g-5} \sum_{j=k+2}^{g-3} \sum_{h=j+2}^{g-1}\left(k j h^{2}\right) \\
& +24 \cdot \sum_{k=1}^{g-4}(k(k+1)(k+2)(k+3))+24 \cdot \sum_{k=1}^{g-5} \sum_{j=k+4}^{g-1}(k(k+1)(k+2) j) \\
& +24 \sum_{k=1}^{g-5} \sum_{j=k+3}^{g-2}(k(k+1) j(j+1))+24 \sum_{k=1}^{g-6} \sum_{j=k+3}^{g-3} \sum_{h=j+2}^{g-1}(k(k+1) j h) \\
& +24 \cdot \sum_{k=1}^{g-5} \sum_{j=k+2}^{g-3}(k j(j+1)(j+2))+24 \cdot \sum_{k=1}^{g-6} \sum_{j=k+2}^{g-4} \sum_{h=j+2}^{g-1}(k j(j+1) h) \\
& +24 \cdot \sum_{k=1}^{g-6} \sum_{j=k+2}^{g-4} \sum_{h=j+2}^{g-2}(k j h(h+1))+24 \cdot \sum_{k=1}^{g-7} \sum_{j=k+2}^{g-5} \sum_{h=j+2}^{g-3} \sum_{=h+2}^{g-1}(k j h f) .
\end{aligned}
$$

The mean values given in the right-hand side of $(1.45) \sim(1.4 .8)$ can be classified into the following types:
(1) $\left(k_{1}^{\prime 1}\right)$
$\left(2^{\circ}\right)\left(k^{l_{1}}(k+1)^{l_{2}}\right)$
$\left(4^{\circ}\right)\left(k^{l_{1}}(k+1)^{l_{2}}(k+2)^{l_{3}}\right)$
$\left(3^{\circ}\right)\left(k^{l^{1}} j^{l_{2}}\right)(j \geqq k+2)$
$\left(6^{\circ}\right)\left(k^{1_{1}} j^{l_{2}}(j+1)^{l_{3}}\right)$
$\left(5^{\circ}\right)\left(k^{l_{1}}(k+1)^{l_{2}} j^{l_{3}}\right)$
(8) $\left(k^{l_{1}}(k+1)^{l_{2}}(k+2)^{l_{3}}(k+3)^{l_{4}}\right)$
$\left(7^{\circ}\right)(k j h)$
$\left(10^{\circ}\right)\left(k^{\prime} j^{\prime 2}(j+1)^{l_{3}}(j+2)^{l_{4}}\right)$
(9) $\left(k^{l_{1}}(k+1)^{l_{2}}(k+2)^{l_{3}} j^{l_{4}}\right)$
(12) $\left(k^{l_{1}}(k+1)^{l_{2}} j^{l_{3}} h^{l_{1}}\right)$

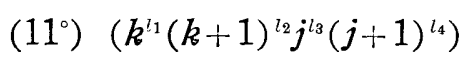
(14 $)\left(k^{1 l} j^{l_{2}} h^{l_{3}}(h+1)^{l_{4}}\right)$

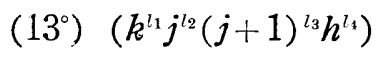
(15) $\left(k^{l_{1}} j^{l_{2}} h^{l_{3}} f^{l_{4}}\right)$,

where we assume $j \geqq k+2, h \geqq j+2$ and $f \geqq h+2$.

1.8. In order to evaluate the mean values given in $\S 1.7$, let us introduce various notations which will be convenient in dealing with them. Let us 
define for each population $\Pi_{i}(i=1,2)$ the probability density element

$$
\begin{aligned}
& \operatorname{Pr} .\left\{x_{1}<\bar{y}_{(k+1)}<d x_{1}, x_{2}<\bar{y}_{\left(k_{2}\right)}<x_{2}+d x_{2}, \ldots, x_{h}<\bar{y}_{\left(k_{h}\right)}<x_{h}+d x_{h} ; \Pi_{i}\right\} \\
& \equiv p^{(i)}\left(\begin{array}{llll}
x_{1} & x_{2} & \ldots & x_{h} \\
k_{1} & k_{2} & \ldots & k_{h}
\end{array}\right) d x_{1} d x_{2} \ldots d x_{h},
\end{aligned}
$$

and then define, for $0<u_{1}, u_{2}, \ldots, u_{h}<\infty$,

$$
\begin{gathered}
p^{(12)}\left(\begin{array}{cccc}
\delta_{1} u_{1} & \delta_{2} u_{2} & \ldots & \delta_{h} u_{h} \\
k_{1} & k_{2} & \ldots & k_{h}
\end{array}\right) \\
\equiv \int_{-\infty}^{\infty} \int_{-\infty}^{\infty} \ldots \int_{1 \infty}^{\infty} p^{(1)}\left(\begin{array}{ccccc}
x_{1}+{ }_{1} u_{1} & x_{2}+{ }_{2} u_{2} & \ldots & x_{h}+{ }_{h} u_{h} \\
k_{1} & k_{2} & \ldots & k_{h}
\end{array}\right) \\
p^{(2)}\left(\begin{array}{ccccc}
x_{1} & x_{2} & \ldots & x_{h} \\
k_{1} & k_{2} & \ldots & k_{h}
\end{array}\right) d x_{1} d x_{2} \ldots d x_{h},
\end{gathered}
$$

where each $\delta_{i}$ can take either 1 or -1 .

By the decomposition given in $\S 1.7$, it is now sufficient to evaluate the integrals of the types given in $\left(1^{\circ}\right) \sim\left(15^{\circ}\right)$ in $\S 1.7$.

Let us write for the sake of convenience the summation over all the possible combinations of the values $\delta_{1}, \delta_{2}, \ldots, \delta_{i k}$ by $\left(\delta_{1} \delta_{2} \ldots \delta_{h}\right)$ and let us denote $d u_{1} d u_{2} \ldots d u_{h}=d^{h} u$. Then we have

$$
\begin{aligned}
& E\left\{S_{k}^{L_{1}}\right\}=\sum_{\left(\delta_{1} \delta_{2}\right)} \int_{0}^{\infty} \int_{0}^{\infty} S_{k}^{l} p^{(12)}\left(\begin{array}{cc}
\delta_{1} u_{1} & \delta_{2} u_{2} \\
k & k+1
\end{array}\right) d^{2} u \\
& E\left\{S_{k}^{l_{1}} S_{k+1}^{l_{2}}\right\}=\sum_{\left(01 \delta_{2} \delta_{3}\right)} \int_{0}^{\infty} \cdot \cdot \int_{0}^{\infty} S_{k}^{L_{1}} S_{k+1}^{l_{2}} \quad p^{(12)}\left(\begin{array}{ccc}
\delta_{1} u_{1} & \delta_{2} u_{2} & \delta_{3} u_{3} \\
k & k+1 & k+2
\end{array}\right) d^{3} u
\end{aligned}
$$

$$
E\left\{S_{k}^{h_{1}} S_{j}^{L_{2}}\right\}=\sum_{\left(\delta 1 \cdots \delta_{4}\right)} \int_{1}^{\infty} . \cdot \int_{0}^{\infty} S_{k}^{L_{1}} S_{j}^{L_{2}} p^{(12)}\left(\begin{array}{llll}
\delta_{1} u_{1} & \delta_{2} u_{2} & \delta_{3} u_{3} & \delta_{4} u_{4} \\
k & k+1 & j & j+1
\end{array}\right) d^{4} u
$$

$$
E\left\{S_{k}^{l_{1}} S_{k+1}^{l_{2}} S_{k+2}^{l_{4}}\right\}=\underset{\left(\delta_{1} \cdots \delta_{4}\right)}{=} \int_{0}^{\infty} . . \int_{0}^{\infty} S_{k}^{l_{1}} S_{k+1}^{l_{2}} S_{k+2}^{l_{3}} p^{(122)}\left(\begin{array}{cccc}
\delta_{1} u_{1} & \delta_{2} u_{2} & \delta_{3} u_{3} & \delta_{4} u_{4} \\
k & k+1 & k+2 & k+3
\end{array}\right) d^{4} u
$$

$$
E\left\{S_{k}^{l_{1}} S_{k+1}^{l_{2}} S_{j}^{l_{3}}\right\}=\sum_{\left(\delta_{1}-\delta_{5}\right)} \int_{0}^{\infty} \ldots \int_{0}^{\infty} S_{k}^{l_{1}} S_{k+1}^{l_{2}} S_{j}^{l_{3}} \quad p^{(12)}\left(\begin{array}{ccccc}
\delta_{1} u_{1} & \delta_{2} u_{2} & \delta_{3} u_{3} & \delta_{4} u_{4} & \delta_{5} u_{5} \\
k & k+1 & k+2 & j & j+1
\end{array}\right) d^{5} u
$$$$
E\left\{S_{k}{ }^{1} S_{j}^{l_{2}} S_{j+1}^{t_{3}}\right\}=\sum_{\left(\delta_{1} \cdots \delta_{5}\right)} \int_{0}^{\infty} \ldots \int_{0}^{\infty} S_{k}^{l_{1}} S_{j}^{l_{2}} S_{j+1}^{l_{3}} \quad p^{(12)}\left(\begin{array}{ccccc}
\delta_{1} u_{1} & \delta_{2} u_{2} & \delta_{3} u_{3} & \delta_{4} u_{4} & \delta_{5} u_{5} \\
k & k+1 & j & j+1 & j+2
\end{array}\right) d^{5} u
$$

$$
E\left\{S_{\Lambda}^{l_{1}} S_{j}^{l_{2}} S_{h}^{l_{3}}\right\}=\underset{\left(\delta_{1}+\delta_{8}\right)}{=\sum} \int_{0}^{\infty} . \cdot \int_{0}^{\infty} S_{k}^{l_{1}} S_{j}^{l_{2}} S_{h}^{l_{3}} \quad p^{(12)}\left(\begin{array}{cccccc}
\delta_{1} u_{1} & \delta_{2} u_{2} & \delta_{3} u_{3} & \delta_{4} u_{4} & \delta_{5} u_{5} & \delta_{6} u_{6} \\
k & k+1 & j & j+1 & h & h+1
\end{array}\right) d^{6} u
$$

$E\left\{S_{k}^{l_{1}} S_{k+1}^{l_{2}} S_{k+2}^{L_{3}} S_{k+3}^{l_{4}}\right\}$

$$
=\sum_{\left(\delta_{1}+\delta_{5}\right)} \int_{0}^{\infty} \int_{0}^{\infty} . \int_{0}^{\infty} S_{k}^{l_{1}} S_{k+1}^{l_{2}} S_{k+2}^{l_{3}} S_{k+3}^{4} p^{(12)}\left(\begin{array}{ccccc}
\delta_{1} u_{1} & \delta_{2} u_{2} & \delta_{3} u_{3} & \delta_{4} u_{4} & \delta_{5} u_{5} \\
k & k+1 & k+2 & k+3 & k+4
\end{array}\right) d^{5} u
$$


$\left(9^{\circ}\right) \quad E\left\{S_{k}^{l_{1}} S_{k+1}^{l_{2}} S_{k+2}^{l_{3}} S_{j}^{l_{1}}\right\}$

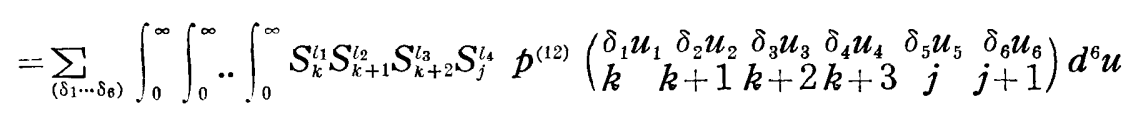

$\left(10^{\circ}\right) \quad E\left\{S_{k}^{l_{1}} S_{j}^{l_{2}} S_{j+1}^{l_{3}} S_{j+2}^{l_{4}}\right\}$

$$
=\sum_{\left(\delta_{1} \cdots \delta_{\theta}\right)} \int_{0}^{\infty} . . \int_{0}^{\infty} S_{k}^{l_{1}} S_{j}^{l_{2}} S_{j+1}^{l_{3}} S_{j+2}^{l_{4}} \quad p^{(12)}\left(\begin{array}{cccccc}
\delta_{1} u_{1} & \delta_{2} u_{2} & \delta_{3} u_{3} & \delta_{4} u_{4} & \delta_{5} u_{5} & \delta_{6} u_{6} \\
k & k+1 & j & j+1 & j+2 & j+3
\end{array}\right) d^{6} u
$$

$\left(11^{\circ}\right) \quad E\left\{S_{k}^{l_{1}} S_{k+1}^{l_{2}} S_{j}^{l_{3}} S_{j+1}^{l_{4}}\right\}$

$$
=\sum_{\left(\delta_{1} \cdots \delta_{6}\right)} \int_{0}^{\infty} . . \int_{0}^{\infty} S_{k}^{l_{1}} S_{k+1}^{l_{2}} S_{j}^{l_{3}} S_{j+1}^{l_{4}} \cdot p^{(12)}\left(\begin{array}{llllll}
\delta_{1} u_{1} & \delta_{2} u_{2} & \delta_{3} u_{3} & \delta_{4} u_{4} & \delta_{5} u_{5} & \delta_{6} u_{6} \\
k & k+1 & k+2 & j & j+1 & j+2
\end{array}\right) d^{6} u
$$

$\left(12^{\circ}\right) \quad E\left\{S_{k}^{l_{1}} S_{(k+1)}^{l_{2}} S_{j}^{l_{3}} S_{h}^{l_{4}}\right\}$

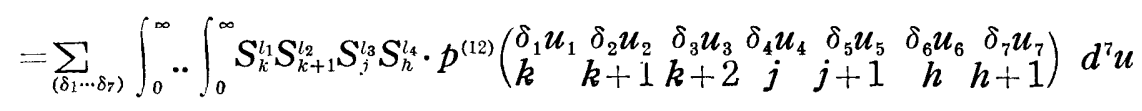

$\left(13^{\circ}\right) \quad E\left\{S_{k}^{t_{1}} S_{j}^{t_{2}} S_{(j+1)}^{t_{3}} S_{h}^{t_{4}}\right\}$

$$
=\sum_{\left(\delta_{1} \cdots \delta_{7}\right)} \int_{0}^{\infty} . \cdot \int_{0}^{\infty} S_{k}^{l_{1}} S_{j}^{l_{2}} S_{j+1}^{l_{3}} S_{l}^{l_{4}} \cdot p^{(12)}\left(\begin{array}{ccccccc}
\delta_{1} u_{1} & \delta_{2} u_{2} & \delta_{3} u_{3} & \delta_{4} u_{4} & \delta_{5} u_{5} & \delta_{6} u_{6} & \delta_{7} u_{7} \\
k & k+1 & j & j+1 & j+2 & h & h+1
\end{array}\right) d^{7} u
$$

$\left(14^{\circ}\right) \quad E\left\{S_{k}^{l_{1}} S_{j}^{l_{2}} S_{h}^{l_{3}} S_{(h+1)}^{l_{4}}\right\}$

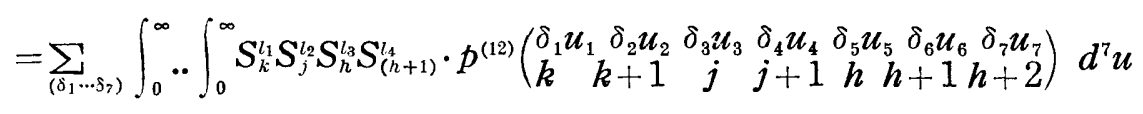

$\left(15^{\circ}\right) \quad E\left\{S_{k}^{l_{1}} S_{j}^{l_{2}} S_{h}^{l_{3}} S_{f}^{l_{4}}\right\}$

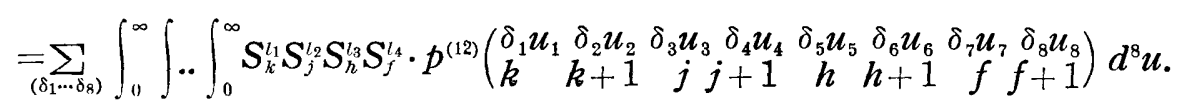

The comblications happen from the fact that

$$
\begin{aligned}
& S_{k}=u_{1}+u_{2} \quad \text { when } \quad \delta_{1} \delta_{2}>0 \\
& =\frac{u_{1}^{2}+u_{2}^{2}}{u_{1}+u_{2}} \quad \text { when } \quad \delta_{1} \delta_{2}<0
\end{aligned}
$$

and similarly for $S_{j}, S_{h}$ and $S_{f}$.

This fact makes it necessary to subdivide each of the classes $\left(1^{\circ}\right) \sim\left(15^{\circ}\right)$ into subclasses according to the sign change of the sequences $\left(\delta_{1} \delta_{2}\right),\left(\delta_{1} \delta_{2} \delta_{3}\right)$, $\ldots$, and $\left(\delta_{1} \delta_{2} \ldots \delta_{8}\right)$.

For instance we have 


$$
\begin{aligned}
& \int_{0}^{\infty} \int_{0}^{\infty} \int_{0}^{\infty} S_{k}^{l_{1}} S_{k+1}^{l_{2}} p^{(12)}\left(\begin{array}{ccc}
u_{1} & u_{2} & u_{3} \\
k & k+1 & k+2
\end{array}\right) d^{3} u \\
& =\int_{0}^{\infty} \int_{0}^{\infty} \int_{0}^{\infty}\left(u_{1}+u_{2}\right)^{l_{1}}\left(u_{2}+u_{3}\right)^{l_{2}} p^{(12)}\left(\begin{array}{ccc}
u_{1} & u_{2} & u_{3} \\
k & k+1 & k+2
\end{array}\right) d^{3} u \\
& \int_{0}^{\infty} \int_{0}^{\infty} \int_{0}^{\infty} S_{k}^{l_{1}} S_{k+1}^{l_{2}} p^{(12)}\left(\begin{array}{ccc}
u_{1} & -u_{2} & u_{3} \\
k & k+1 & k+2
\end{array}\right) d^{3} u \\
& =\int_{0}^{\infty} \int_{0}^{\infty} \int_{0}^{\infty}\left(\frac{u_{1}^{2}+u_{2}^{2}}{u_{1}+u_{2}}\right)^{l_{1}}\left(\frac{u_{2}^{2}+u_{3}^{2}}{u_{2}+u_{3}}\right)^{l_{2}} p^{(12)}\left(\begin{array}{ccc}
u_{1} & -u_{2} & u_{3} \\
k & k+1 & k+2
\end{array}\right) d^{3} u \\
& \int_{0}^{\infty} \int_{0}^{\infty} \int_{0}^{\infty} S_{k}^{l_{1}} S_{k+1}^{l_{2}} \quad p^{(12)}\left(\begin{array}{lll}
u_{1} & -u_{2}-u_{3} \\
k & k+1 & k+2
\end{array}\right) d^{3} u \\
& =\int_{0}^{\infty} \int_{0}^{\infty} \int_{0}^{\infty}\left(\frac{u_{1}^{2}+u_{2}^{2}}{u_{1}+u_{2}}\right)^{l_{1}}\left(u_{2}+u_{3}\right)^{l_{2}} p^{(12)}\left(\begin{array}{lll}
u_{1} & -u_{2} & -u_{3} \\
k & k+1 & k+2
\end{array}\right) d^{3} u \\
& \int_{0}^{\infty} \int_{0}^{\infty} \int_{0}^{\infty} \int_{0}^{\infty} S_{k}^{l_{1}} S_{h}^{l_{2}} \quad p^{(12)}\left(\begin{array}{cccc}
u_{1} & u_{2} & -u_{3} & -u_{4} \\
k & k+1 & h & h+1
\end{array}\right) d^{4} u \\
& =\int_{0}^{\infty} \int_{0}^{\infty} \int_{0}^{\infty} \int_{0}^{\infty}\left(u_{1}+u_{2}\right)^{l_{3}}\left(\frac{u_{3}^{2}+u_{4}^{2}}{u_{3}+u_{4}}\right)^{l_{2}} p^{(12)}\left(\begin{array}{cccc}
u_{1} & u_{2} & -u_{3} & -u_{4} \\
k & k+1 & h & h+1
\end{array}\right) d^{4} u
\end{aligned}
$$

and similarly for other summands given above.

§. The asymptotic form of the multivariate probability density function of $\left(\overline{\boldsymbol{y}}_{\left(k_{1}\right),}, \overline{\boldsymbol{y}}_{\left(\left(k_{2}\right),\right.}, \ldots, \overline{\boldsymbol{y}}_{\left.\left(k_{h}\right)\right)}\right)$

2.1. In order to derive the asymptotic form of the probability density function $p\left(u_{1}, u_{2}, \ldots, u_{k}\right)$ and $p^{(12)}\left(u_{1}, u_{2}, \ldots, u_{k}\right)$ we shall appeal to the two asymptotic properties valid under their respective conditions. For the sake of convenience we use

Definition 2.1. Let $\left\{X_{1}, X_{2}, \ldots, X_{k}\right\}$ and $\left\{X_{1}^{(n)}, X_{2}^{(n)}, \ldots, X_{k}^{(n)}\right\}(n=1,2, \ldots)$ be a sequence of $k$-dimensional muItivariate stochastic variables, and let it be assumed that for any assigned set of $k$ real numbers $\left(u_{1}, u_{2}, \cdots, u_{k}\right)$

(1') $\operatorname{Pr} .\left\{X_{1}^{(n)} \leqq u_{1}, X_{2}^{(n)} \leqq u_{2}, \ldots, X_{k}^{(n)} \leqq u_{k}\right\}$

$$
\equiv F_{n}\left(u_{1}, u_{2}, \ldots, u_{k}\right)=\int_{-\infty}^{u_{1}} \int_{-\infty}^{u_{2}} \ldots \int_{-\infty}^{u_{k}} f_{n}\left(t_{1}, t_{2}, \ldots, t_{k}\right) d t_{1} d t_{2} \ldots d t_{k}
$$

$$
\begin{aligned}
& \text { (2) } \quad \operatorname{Pr} .\left\{X_{1} \leqq u_{1}, X_{2} \leqq u_{2}, \ldots, X_{k} \leqq u_{k}\right\} \\
& \equiv F\left(u_{1}, u_{2}, \ldots, u_{k}\right)=\int_{-\infty}^{u_{1}} \int_{-\infty}^{u_{2}} \ldots \int_{-\infty}^{u_{k}} f\left(t_{1}, t_{2}, \ldots, t_{n}\right) d t_{1} d t_{2} \ldots d t_{n}
\end{aligned}
$$


(3) $\lim _{n \rightarrow \infty} f_{n}\left(u_{1}, u_{2}, \ldots, u_{k}\right)=f\left(u_{1}, u_{2}, \ldots, u_{k}\right)$.

Then the probability density function is said to be asymptotically convergent in probability law to the probability density function $f\left(\boldsymbol{u}_{1}, \boldsymbol{u}_{2}, \ldots, \boldsymbol{u}_{k}\right)$ and is denoted by

$$
f_{n}\left(u_{1}, u_{2}, \ldots, u_{k}\right) \stackrel{\left(n^{\prime}\right)}{\cong} f\left(u_{1}, u_{2}, \ldots, u_{k}\right) .
$$

2.2. The first asymptotic property is concerned with the asymptotic convergence in law of the probability density function $f_{t_{1}}{ }^{*} f_{t_{2}}{ }^{*} \ldots f_{t_{n^{\prime}}}\left(\boldsymbol{n}^{\prime} u\right)$ to the normal probability density. Indeed we shall make

Assumption I. For each assigned value of $\left(t_{1}, t_{2}, \ldots, t_{n^{\prime}}\right)$ we have

$$
\begin{aligned}
& f_{t_{1}}{ }^{*} f_{t_{2}}{ }^{*} f_{t_{n^{\prime}}}\left(n^{\prime} u\right) \\
& \stackrel{\left(n^{\prime}\right)}{\cong} \frac{1}{\sqrt{2 \pi \sigma_{2}^{2}\left(1-\rho^{2}\right) n^{\prime}}} \exp \left\{-\begin{array}{c}
n^{\prime}\left(u-\left(\mu_{2}+\rho \sigma_{1} \sigma_{2}^{-1} \bar{t}\right)\right)^{2} \\
2 \sigma_{2}^{2}\left(1-\rho^{2}\right)
\end{array}\right\},
\end{aligned}
$$

where

$$
\bar{t}=\left(t_{1}+t_{2}+\ldots+t_{n^{\prime}}\right) / \boldsymbol{n}^{\prime} .
$$

The practical uses of this assumption are not only concerned with large $n^{\prime}$, but also with moderate size of $\boldsymbol{n}^{\prime}$, as may be expected from the central limit theorem.

Example 2.1. Let us consider the bivariate normal population or populations of the type

$$
f(x, y)=\frac{1}{2 \pi \sigma_{1} \sigma_{2}\left(1-\rho^{2}\right)^{1 / 2}} \exp \{-Q\},
$$

where

$$
Q \equiv \frac{1}{2\left(1-\rho^{2}\right)}\left(\frac{\left(x-\mu_{1}\right)^{2}}{\sigma_{1}^{2}}-2 \rho \frac{\left(x \mu-{ }_{1}\right)\left(y-\mu_{2}\right)}{\sigma_{1} \sigma_{2}}+\frac{\left(y-\mu_{2}\right)^{2}}{\sigma_{2}^{2}}\right) .
$$

Then the marginal probability density function of $x$ and the conditional probability density function of $y$ for an assigned $x$ are given by

$$
\begin{aligned}
& f_{1}(x)=\frac{1}{\sqrt{2 \pi \sigma_{1}^{2}}} \exp \left\{-\frac{\left(x-\mu_{1}\right)^{2}}{2 \sigma_{1}^{2}}\right\} \\
& f\left(y^{\prime} x\right)=\frac{1}{\sqrt{2 \pi \sigma_{2}^{2}\left(1-\rho^{2}\right)}} \exp \left\{-\frac{\left(y-\mu_{2}-\left(\rho \sigma_{2} / \sigma_{1}\right)\left(x-\mu_{1}\right)\right)^{2}}{2 \sigma_{2}^{2}\left(1-\rho^{2}\right)}\right\} .
\end{aligned}
$$

Consequently we have, in view of (1.08),

$$
\begin{aligned}
& E\left\{\boldsymbol{y}_{(k i))}^{l}\right\} \\
= & \frac{n !}{(k-1) !(n-k) !} \int_{-\infty}^{\infty}\left(F_{1}(x)\right)^{k-1} f_{1}(x)\left(1-F_{1}(x)\right)^{n-k}\left(\int_{-\infty}^{\infty} y^{l} f(y \mid x) d y\right) d x
\end{aligned}
$$




$$
\begin{aligned}
= & \frac{n !}{(k-1) !(n-k) !} \int_{-\infty}^{\infty}\left(F_{1}(x)\right)^{k-1} f_{1}(x)\left(1-F_{1}(x)\right)^{n-k} \\
& \cdot \frac{1}{\sqrt{ }} \frac{1}{2 \pi} \int_{-\infty}^{\infty}\left(\mu_{2}+\rho \frac{\sigma_{2}}{\sigma_{1}}\left(x-\mu_{1}\right)+\sigma_{2}\left(1-\rho^{2}\right)^{1 / 2} \tau\right)^{l} e^{-\tau^{3 / 2}} d \tau \\
= & \frac{n !}{(k-1) !(n-k) !} \int_{-\infty}^{\infty}\left(F_{1}(x)\right)^{k-1} f_{1}(x)\left(1-F_{1}(x)\right)^{n-k} \\
& \cdot \sum_{k=0}^{l}{ }_{l} C_{h}\left(\mu_{2}+\rho \frac{\sigma_{2}}{\sigma_{1}}\left(x-\mu_{1}\right)\right)^{l-h} \sigma_{2}^{n}\left(1-\rho^{2}\right)^{n / 2} E_{1}\left\{\tau^{n}\right\},
\end{aligned}
$$

where $E_{1}\left\{\tau^{h}\right\}$ are equal to zero for odd positive integers $h$, while for even positive integers $h$ we have

$$
E_{1}\left\{\tau^{h}\right\}=1.3 .5 \ldots \ldots(h-1),
$$

and $E_{1}\left\{\tau^{h}\right\}=1$.

The transformation $t=\left(x-\mu_{1}\right) / \sigma_{1}$ yields us therefore

$$
\begin{gathered}
E\left\{\boldsymbol{y}_{(k k)}^{l}\right\}=\frac{n !}{(k-1) !(n-k) !} \int_{-\infty}^{\infty}(\Phi(t))^{k-1} \varphi(t)(1-\Phi(t))^{n-k} \\
\cdot\left\{\sum_{h=0}^{l}{ }_{l} C_{h}\left(\mu_{2}+\rho \sigma_{2} t\right)^{l-h} \sigma_{2}^{h}\left(1-\rho^{2}\right)^{h / 2} E_{1}\left\{\tau^{h}\right\}\right\} d t,
\end{gathered}
$$

where we have put

$$
\begin{aligned}
& \varphi(t)=\frac{1}{\sqrt{2 \pi}} e^{-t 2 / 2} \\
& \Phi(t)=\int_{-\infty}^{t} \varphi(u) d u .
\end{aligned}
$$

The special case when $\mu_{2}=0$ may be worth while for us to mention. Indeed we have then

$$
E\left\{y_{(k))}^{l}\right\}=\sigma_{2}^{l} \rho^{l} \sum_{h=0}^{l}{ }_{l} C_{h}\left(\frac{1-\rho^{2}}{\rho^{2}}\right)^{h / 2} E_{1}\left\{\tau^{h}\right\} E\left\{\sigma_{(k)}^{l-h}\right\},
$$

where we have put

$$
E\left\{X_{(k)}^{s}\right\} \equiv \frac{n !}{(k-1) !(n-k) !} \int_{-\infty}^{\infty}(\Phi(t))^{k-1} t^{s} \varphi(t)(1-\Phi(t))^{n-k} d t .
$$

The similar argument gives us that, for $i<j$, in view of (1.09), (2.15) $E\left\{\boldsymbol{y}_{(i))}^{l} y_{(j))}^{m}\right\}$ 


$$
\begin{gathered}
=\frac{n !}{(i-1) !(j-i-1) !(n-j) !} \int_{-\infty}^{\infty} d x_{1} \int_{x_{1}}^{\infty}\left(F_{1}\left(x_{1}\right)\right)^{i-1} f_{1}\left(x_{1}\right)\left(F_{1}\left(x_{2}\right)-F_{1}\left(x_{1}\right)\right)^{j-i-1} \\
f_{1}\left(x_{2}\right)\left(1-F_{1}\left(x_{2}\right)\right)^{n-j} d x_{2} \\
\cdot \int_{-\infty}^{\infty} y_{1}^{\prime} f\left(y_{1} \mid x_{1}\right) d y_{1} \int_{-\infty}^{\infty} y_{2}^{m} f\left(y_{2} x_{2}\right) d y_{2} \\
=\frac{n !}{(i-1) !(j-i-1) !(n-j) !} \int_{-\infty}^{\infty} d x_{1} \int_{x_{1}}^{\infty}\left(F_{1}\left(x_{1}\right)\right)^{i-1} f_{1}\left(x_{1}\right)\left(F_{1}\left(x_{2}\right)-F_{1}\left(x_{1}\right)\right)^{j-i-1} \\
\quad \sum_{1}^{l}\left(x_{2}\right)\left(1-F_{1}\left(x_{2}\right)\right)^{n-j} d x_{2} \\
\cdot \sum_{g=0}^{m} C_{m} C_{g}\left(\mu_{2}+\rho \frac{\sigma_{2}}{\sigma_{1}}\left(x_{1}-\mu_{1}\right)^{l-h} \sigma_{2}^{h}\left(1-\rho^{2}\right)^{n / 2} E_{1}\left\{\tau^{n}\right\}\right.
\end{gathered}
$$

In particular for $\mu_{2}=0$, we have

$$
=\sigma_{2}^{l+m} \rho^{l+m} \sum_{h=0}^{l} \sum_{g=0}^{m}{ }_{l} C_{h} \cdot{ }_{m} C_{o}\left(\frac{1-\rho^{2}}{\rho^{2}}\right)^{\frac{n+q}{2}} E_{1}\left\{\tau^{h}\right\} E_{1}\left\{\tau^{g}\right\} E\left\{X_{(i)}^{l-h} X_{(j)}^{m-g}\right\},
$$

where

$$
\begin{gathered}
=\frac{n !}{(i-1) !(j-i-1) !(n-j) !} \int_{-\infty}^{\infty}\left(\Phi\left(t_{1}\right)\right)^{i-1} t_{1}^{s} \varphi\left(t_{1}\right) d t_{1} \\
\cdot \int_{t_{1}}^{\infty}\left(\Phi\left(t_{2}\right)-\Phi\left(t_{1}\right)\right)^{j-i-1} t_{2}^{q} \varphi\left(t_{2}\right)\left(1-\Phi\left(t_{2}\right)\right)^{n-j} d t_{2} .
\end{gathered}
$$

Our results (2.10) and (2.16) show us that $E\left\{y_{(k)\rangle}^{l}\right\}$ and $E\left\{\boldsymbol{y}_{(i)\rangle}^{l} \boldsymbol{y}_{(j)\rangle}^{m}\right\}$ can be written in term of the constants associated with the order statistics defined for the standard normal population which have been fully discussed by various authors.

We have also

$$
\begin{aligned}
& f_{t_{1}}{ }^{*} f_{t_{2}}{ }^{*} \ldots f_{\boldsymbol{u}^{\prime} n}\left(\boldsymbol{n}^{\prime} \boldsymbol{u}\right) \\
& \quad=\frac{1}{\sqrt{ } / \overline{2 \pi \boldsymbol{n}^{\prime} \sigma_{2}^{2}\left(1-\rho^{2}\right)}} \exp \left\{-\frac{\left(\boldsymbol{u}-\left(\mu_{2}+\rho \frac{\sigma_{2}}{\sigma_{1}}\left(\bar{t}-\mu_{1}\right)\right)^{2}\right.}{2 \boldsymbol{n}^{\prime} \sigma_{2}^{2}\left(1-\rho^{2}\right) .}\right\} .
\end{aligned}
$$

Consequently we have

$$
\operatorname{Pr}_{.}\left\{u<\boldsymbol{y}_{(k))}<u+d u\right\} \equiv h_{k}(u) d u
$$




$$
\begin{aligned}
& =\frac{n ! d u}{\left((k-1) n^{\prime}\right) ! n^{\prime} !\left((g-k) n^{\prime}\right) !} \\
& \cdot \int_{-\infty<t_{1}<t_{2}<\cdots<t_{n^{\prime}}<\infty} \int_{1} \ldots \int_{i=1}\left(\Phi\left(t_{1}\right)\right)^{(k-1) n^{\prime}}\left(1-\Phi\left(t_{n^{\prime}}\right)\right)^{(g-k) n^{\prime}} \prod_{i=1}^{n^{\prime}} \varphi\left(t_{i}\right) \\
& \quad \frac{n^{\prime 1 / 2}}{\left(2 \pi \sigma_{2}^{2}\left(1-\rho^{2}\right)\right)^{1 / 2}-\varphi\left(\frac{n^{\prime 1 / 2}\left(u-\left(\mu_{2}+\rho \sigma_{2} \bar{t}\right)\right)}{\sigma_{2}\left(\overline{1}-\rho^{2}\right)^{1 / 2}}\right) d t_{1} \ldots d t_{n^{\prime}},}
\end{aligned}
$$

and similarly

$$
\begin{aligned}
& \operatorname{Pr} .\left\{u<\bar{y}_{(k))}<u+d u, \quad v<\bar{y}_{(k+1))}<v+d v\right\} \equiv p_{k}(u, v) d u d v \\
& =\frac{n ! d u}{((k-1)} \frac{n}{\left.n^{\prime}\right) ! n^{\prime} ! N^{\prime} !((g-k-1)} \overline{\left.n^{\prime}\right) !}
\end{aligned}
$$

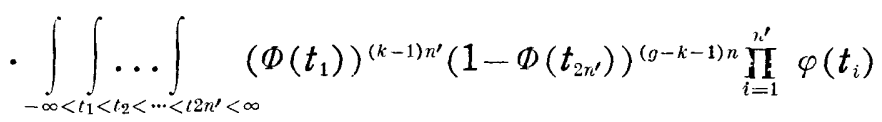

$$
\begin{aligned}
& \frac{\boldsymbol{n}^{\prime 1 / 2}}{\left(2 \pi \sigma_{2}^{2}\left(1-\rho^{2}\right)\right)^{1 / 2}} \varphi\left(\frac{\boldsymbol{n}^{\prime}\left(\boldsymbol{u}-\left(\mu_{2}+\rho \sigma_{2} \bar{t}_{1}\right)\right)}{\sigma_{2}\left(1-\rho^{2}\right)^{1 / 2}}\right) d t_{1} \ldots d t_{n^{\prime}} \\
& \frac{n^{\prime / 2}}{\left(2 \pi \sigma_{2}^{2}\left(1-\rho^{2}\right)\right)^{1 / 2}} \varphi\left(\frac{n^{\prime}\left(v-\left(\mu_{2}+\rho \sigma_{2} \bar{t}_{2}\right)\right)}{\sigma_{2}\left(1-\rho^{2}\right)^{1 / 2}}\right) d t_{n+1} \ldots d t_{2 n^{\prime}},
\end{aligned}
$$

where we have put

$$
\bar{t}_{k}=\left(t_{1}+t_{2}+\ldots+t_{n^{\prime}}\right) / n^{\prime} ; \bar{t}_{k+1}=\left(t_{n^{\prime}+1}+\ldots+t_{2 n^{\prime}}\right) / n^{\prime} .
$$

Turning back to our general situation, let us consider the means of $n^{\prime}$ successive order statistics

$$
\boldsymbol{x}_{(k)}=\left(\boldsymbol{x}_{\left((k-1) n^{\prime}+1\right)}+\boldsymbol{x}_{\left((k-1) n^{\prime}+2\right)}+\ldots+\boldsymbol{x}_{\left(k n^{\prime}\right)}\right) / \boldsymbol{n}^{\prime}
$$

for $k=1,2, \ldots, g-1$. Let us denote by

$$
r_{n^{\prime}}\left(\begin{array}{cccc}
t_{1} & t_{2} & \ldots & t_{h} \\
k_{1} & k_{2} & \ldots & k_{h}
\end{array}\right)
$$

the simultaneous probability density function of the statistics $\left(\bar{x}_{\left(k_{1}\right)}, \bar{x}_{\left(k_{2}\right)}, \ldots\right.$, $\left.\bar{x}_{\left(k_{h}\right)}\right)$, that is,

$$
\begin{aligned}
& \operatorname{Pr} .\left\{t_{1}<\bar{x}_{\left(k_{1}\right)}<t_{1}+d t_{1}, t_{2}<\bar{x}_{\left(k_{2}\right)}<t_{2}+d t_{2}, \ldots, t_{h}<\bar{x}_{\left(k^{k} h_{2}\right)}<t_{h}+d t_{h}\right\} \\
& \equiv r_{n^{\prime}}\left(\begin{array}{ccc}
t_{1} t_{2} & \ldots & t_{h} \\
k_{1} k_{2} & \ldots & k_{h}
\end{array}\right) d t_{1} d t_{2} \ldots d t_{h}
\end{aligned}
$$

for any set of $h$ real numbers $\left(t_{1}, t_{2}, \ldots, t_{h}\right)$, where $1 \leq k_{1}<k_{2}<\cdots<k_{h}<g-1$.

Now it is to be noted that under our Assumption I we have

$$
\begin{aligned}
& \operatorname{Pr} .\left\{u_{1}<\bar{y}_{\left(k_{1}\right)}<u_{1}+d u_{1}, u_{2}<\bar{y}_{\left.\left(k_{2}\right)\right)}<u_{2}+d u_{2}, \ldots, u_{h}<\bar{y}_{\left(k_{h}\right)}<u_{h}+d u_{h}\right\}
\end{aligned}
$$

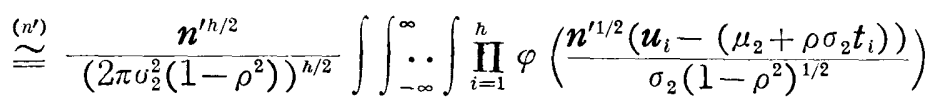




$$
\boldsymbol{r}_{n^{\prime}}\left(\begin{array}{lll}
t_{1} t_{2} & \ldots & t_{h} \\
k_{1} k_{2} & \ldots & k_{h}
\end{array}\right) d t_{1} d t_{2} \ldots d t_{h} .
$$

2.3. The second asymptotic property is to appeal to the asymptotic normality of the order statistic. In what follows let us make the following

Assumption II. The probability density function $f_{1}(x)$ is differentiable in the closure of its carrier $c\left(f_{1}\right)$ and $f_{1}^{\prime}(x)=0$ in the interior of $c\left(f_{1}\right)$.

Under our Assumption II we can appeal to the theorem due to $\mathrm{F}$. MOSTELLER [1].

Theorem 1. (F. MOSTELLER) Let $m$ be any assinged positive integers and let $\left\{\lambda_{i}\right\} \quad(i=1,2, \ldots, m)$ by any assigned set of $\boldsymbol{m}$ fractional numbers $0<\lambda_{1}<\lambda_{2}<<\lambda_{m}<1$. and let the $\lambda_{i}$-quantile of the population be denoted by $\tau_{i}$, i.e.,

$$
F_{1}\left(\tau_{i}\right) \equiv \int_{-\infty}^{\tau_{i}} f_{1}(t) d t=\lambda_{i} \quad(i=1,2, \ldots, m) .
$$

Let $n_{i}=\left[n \lambda_{i}\right]+1, i=1,2, \ldots, m$, where $[x]$ denotes the largest integer not greater than $x$.

Then as $n$ tends infinity the simultaneous probability distribution of the $m$ order statistics $\left(x_{\left(n_{1}\right)}, x_{\left(n_{2}\right)}, \ldots, x_{\left(n_{m}\right)}\right)$ tends to a $k$-dimensional normal distribution with the means $\left(\tau_{1}, \tau_{2}, \ldots, \tau_{m}\right)$ and the variance-covariance $\boldsymbol{m} \times \boldsymbol{m}$ matrix $\left(\lambda_{i}\left(1-\lambda_{j}\right) / n f_{1}\left(\tau_{i}\right) f\left(\tau_{j}\right)\right)(i, j=1,2, \ldots, m)$.

Let us apply this theorem to the approximation of the integral (2.25). Let us consider the set of fractional numbers $\left\{\lambda_{k, i}^{(n)}\right\},\left\{\tau_{k, i}^{(n)}\right\}$ and $\left\{\boldsymbol{g}_{k, i}^{(n)}\right\} \quad(\boldsymbol{k}=1$, $\left.2, \ldots, g-1 ; i=1,2, \ldots, n^{\prime}\right)$ such that

$$
\begin{aligned}
& \lambda_{k, i}^{(n)} \equiv\left((k-1) n^{\prime}+i\right) / n, \\
& F_{1}\left(\tau_{k, i}^{(n)}\right) \equiv \int_{-\infty}^{\tau_{k, i}^{(n)}} f_{1}(t) d t=\lambda_{k, i}^{(n)} \\
& g_{k, i}^{(n)} \equiv f_{1}\left(\tau_{k, i}^{(n)}\right) .
\end{aligned}
$$

The direct application of Mosteller's theorem gives us for any assigned $h, 0<h<g-1$

Lemma 2.2. As $n^{\prime}$ tends to infinity the $h$-dimensional multivariate statistic $\left(\bar{x}_{\left(k_{1}\right)}, \bar{x}_{\left(k_{2}\right)}, \ldots, \bar{x}_{(k h)}\right)$ is asymptotically distributed in the $h$-dimentional normal distribution with the $h$-dimensional mean vector $\left(m_{\left(k_{1}\right)}, m_{\left(k_{2}\right)}, \ldots, m_{\left(k_{h}\right)}\right)$ and the $h \times h$ variance-covariance $\operatorname{matrix}\left(\frac{\left.\sigma_{\left(k_{p}, k_{q}\right)}\right)}{n^{\prime}}\right)(p, q=1,2, \ldots, h)$ such that

$$
\begin{aligned}
& \boldsymbol{m}_{\left(k_{l}\right)} \equiv \frac{\tau_{k_{l}, 1}^{(n)}+\tau_{k_{l}, 2}^{(n)}+\ldots+\tau_{k_{l}, n^{\prime}}^{(n)}}{n^{\prime}}
\end{aligned}
$$

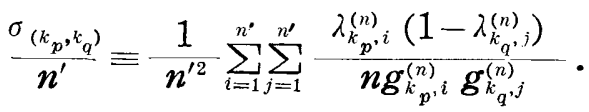


Let us write for the sake of convenience

$$
\begin{aligned}
& \sigma_{\left(k_{p}, k_{p}\right)} \equiv \sigma_{(k)}^{2} \\
& \rho_{\left(k_{p}, k_{q}\right)} \equiv \sigma_{\left(k_{p}, k_{q}\right)} / \sigma_{\left(k_{p}\right)} \sigma_{\left(k_{q}\right)} .
\end{aligned}
$$

2.4. The combination of Lemma 2.1 and 2.2 gives us

Theorem 2.1. Under a situation where both Assumptions I and II hold true, the simultaneous probability density function of $\left(\bar{y}_{\left(k_{1}\right)}, \bar{y}_{\left(k_{i k}\right)}, \ldots, \bar{y}_{\left(k_{n}\right)}\right)$ is asymptotically convergent in probability law, as $n^{\prime}$ tends to infinity, to the $h$-variate normal distribution with the mean vector $\left(\xi_{\left(k_{1}\right),}, \xi_{\left\langle\left(k_{2}\right\rangle\right.}, \ldots, \xi_{\left\langle\left(k_{h}\right)\right.}\right)$ and the $h \times h$ variance-covariance matrix $\left(\rho_{\left(k_{i}, k_{j}\right)} \sigma_{\left(k_{i}\right)} \sigma_{\left(k k_{j}\right)}\right)(i, j=1,2, \ldots, h)$ such that

$$
\begin{aligned}
& \xi_{\left\langle k_{i\rangle}\right)} \equiv \mu_{2}-\rho \boldsymbol{m}_{\left(k_{i}\right)} \sigma_{2} \\
& \sigma_{[(k)}^{2} \equiv \sigma_{\{k, k)} \equiv \sigma_{2}^{2}\left(\frac{1-\rho^{2}}{\boldsymbol{n}_{k}^{\prime}}+\rho^{2} \sigma_{(k)}^{2}\right) \quad(k=1 \sim 5) \\
& \sigma_{\left(k_{1}, k_{2}\right)} \equiv \rho^{2} \sigma_{\left(k_{1}, k_{2}\right)} \quad\left(k_{1} \div k_{i} ; k_{1}, k_{2}=1 \sim 5\right) \\
& \rho_{\left(k_{1}, k_{2}\right)} \equiv \frac{\sigma_{\left(k_{1}, k_{2}\right)}}{\sigma_{\left(k_{1}\right)} \sigma_{\left(k k_{2}\right)}} \quad\left(k_{1}, k_{2}=1 \sim 5\right),
\end{aligned}
$$

where $h$ is subject to the same condition to Lemma $2.2,0<\boldsymbol{h}<\boldsymbol{g}-1$.

Proof: In virtue of Assumption I, we have the asymptotic probability density function given by the right-hand side of (2.25). Now let us write $(2,37)$

$$
\bar{y}_{\left(k_{i}\right)}=\mu_{2}+\rho \sigma_{2} \bar{x}_{\left(k_{i}\right)}+n^{\prime-1 / 2} \sigma_{2}\left(1-\rho^{2}\right)^{1 / 2} z_{k_{i}}
$$

$(i=1,2, \ldots, h)$. Then $(2.25)$ gives us that $\left(1^{\circ}\right)$ the $h$-dimensional variate $\left(z_{k_{1}}, z_{k_{2}}, \ldots, z_{k_{n}}\right)$ is asymptotically convergent in probability law to the $h$-dimensional normal variate with the mean vector $(0,0, \ldots, 0)$ and the variancecovariance matrix which reduces to the unit-matrix, and that $\left(2^{\circ}\right)\left(z_{k_{1}}, z_{k_{2}}\right.$, $\left.\ldots, z_{k_{h}}\right)$ is asymptotically independent to the variate $\left(\bar{x}_{\left(k_{1}\right)}, \bar{x}_{\left(k_{2}\right)}, \ldots, \bar{x}_{\left(k_{k}\right)}\right)$. Consequently we have, as $\boldsymbol{n}^{\prime}$ tends to infinity,

$$
\begin{aligned}
& E\left\{\bar{y}_{\left(k_{i)}\right)}\right\} \cong \mu_{2}+\rho \sigma_{2} \boldsymbol{m}_{k_{i}} \\
& \sigma^{2}\left\{\overline{\boldsymbol{y}}_{\left(k_{i, j}\right.}\right\} \cong \rho^{2} \sigma_{2}^{2} \sigma^{2}\left\{\overline{\boldsymbol{X}}_{\left(k_{i}\right)}\right\}+\boldsymbol{n}^{\prime-1} \sigma_{2}^{2}\left(1-\rho^{2}\right) \sigma^{2}\left\{\boldsymbol{Z}_{i}\right\} \\
& \operatorname{Cov} .\left\{\bar{y}_{\left(k_{i}\right)}, \bar{y}_{\left(k_{j}\right)}\right\} \cong \operatorname{Cov} .\left\{\rho \sigma_{2} \overline{\boldsymbol{x}}_{\left(k_{i}\right)}, \rho \sigma_{2} \overline{\boldsymbol{x}}_{\left(k_{j}\right)}\right\} .
\end{aligned}
$$

Now the application of Lemma 2.2 gives us

$$
\begin{aligned}
& \sigma^{2}\left\{\overline{\boldsymbol{y}}_{\left(\left\langle k_{i}\right)\right.}\right\} \cong \sigma_{2}^{2}\left(\rho^{2} \sigma_{\left(k_{i}\right)}^{2}+\left(1-\rho^{2}\right)\right) / \boldsymbol{n}^{\prime} \\
& \operatorname{Cov}\left\{\bar{y}_{\left\{\left(k_{i}\right)\right.}, \bar{y}_{\left\{k_{j}\right)}\right\} \cong \rho^{2} \sigma_{2}^{2} \rho_{\left(k_{i}, k_{j}\right)} \sigma_{\left(k_{i}\right)} \sigma_{\left(k_{j}\right)}
\end{aligned}
$$

as we were to prove.

Theorem 2.2. Let $\Pi_{1}$ and $\Pi_{2}$ be two bivariate populations from which two random samples $\left\{\left(x_{i}, y_{i}\right)\right\}(i=1.2, \cdots, n)$ and $\left\{\left(x_{i}^{\prime}, z_{i}\right)\right\}(i=1,2, \ldots, n)$ are drawn independently. Let $\left\{\left(x_{(i)}, \boldsymbol{y}_{(i)}\right)\right\}$ and $\left\{\left(x_{(i)}^{\prime}, z_{(i)}\right)\right\}$ be defined as in

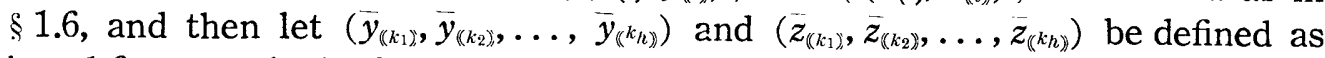
in $\$ 1.6$ respertively for each of these two samples. Let the Assumptions 
I and II be satisfied with their respective values of $\mu_{2}, \rho, \sigma_{1}, \sigma_{2}, \rho_{\left(k_{p}, k_{q}\right)}, \sigma_{\left(k_{p}\right)}$ for each of these two populations, which are denoted by $\mu_{2}^{(i)}, \rho^{(i)}, \sigma_{1}^{(i)}, \boldsymbol{m}_{k_{p}}^{(i)}$, $\sigma_{2}^{(i)} \rho_{\left(k_{p} k_{q}\right)}^{(i)}, \sigma_{\left(k_{p}\right)}^{(i)}$, with $i=1$ for $\Pi_{1}$ and with $i=2$ for $\Pi_{2}$.

Then the simultaneous probability density function of $\left(\bar{y}_{\left.\left\{k_{1}\right\rangle\right)}-\bar{z}_{\left\{k k_{1}\right\}}, \bar{y}_{\left\langle k_{2}\right\rangle}\right.$ $\left.-\overline{\boldsymbol{z}}_{\left\{\left\langle k_{2}\right\}\right.}, \ldots, \bar{y}_{\left(k_{h}\right)}-\overline{\boldsymbol{z}}_{\left\{k_{h}\right)}\right)$ is asymptotically convergent in probability law, as $n^{\prime}$ tend tends to infinity, to the $k$-variate normal distribution with the mean vector $\left(\delta_{\left\langle k_{1}\right\rangle}, \delta_{\left(k_{k 2}\right)}, \ldots, \delta_{\left\{\left(k_{h}\right)\right.}\right)$ and the $h \times h$ variance-covariance matrix $\left(\sigma_{\left(k_{i}, k_{j, j}\right)}^{(1.2)}\right)$ such that

$$
\begin{aligned}
& \delta_{\left(k k_{l}\right)} \equiv\left(\mu_{2}^{(1)}-\rho^{(1)} \boldsymbol{m}_{\left(k_{i}\right)}^{(1)} \sigma_{2}^{(1)}\right)-\left(\mu_{2}^{(2)}-\rho^{(2)} \boldsymbol{m}_{\left(k_{i}\right)}^{(2)} \sigma_{2}^{(2)}\right. \\
& \sigma_{\|\left(k_{i}, \cdot, k_{j}\right)}^{(1)} \equiv \sigma_{\left(\left\langle k_{i}, k_{j}\right)\right.}^{(1)}+\sigma_{\left(k k_{i}, k_{j}\right)}^{(2),},
\end{aligned}
$$

where

$$
(i, j=1,2, \ldots, g-1) \text {. }
$$

$$
\begin{aligned}
& \sigma_{\left(k_{i}, k_{j}\right)}^{(1) 2}=\rho^{(1) 2} \rho_{\left(k_{i}, k_{j}\right)}^{(1)} \sigma_{\left(k_{i}\right)}^{(1)} \sigma_{\left(k_{j}\right)}^{(1)} \\
& \sigma_{\left(k k_{i}, k_{k}\right)}^{(2)}=\rho^{(2) 2} \rho_{\left(k_{i}, k_{j}\right)}^{(2)} \sigma_{\left(k_{i}\right)}^{(2)} \sigma_{\left(k_{j}\right)}^{(2)}
\end{aligned}
$$

Proof: Immediate from Theorem 2.1 and (1.50).

\section{\$3. The evaluation of the fundamental integrals}

3.1. In view of Theorem 2.2 , and the results given in $\S 1.8$, the evaluations of the integrals are reduced to those associated with the multivariate normal populations under the Assumptions I and II, provided that we are interested at least with a fairly large or moderately large $n^{\prime}$. It is the purpose of this paragraph to enunciate a general procedure to evaluate the integrals of the type:

$$
\begin{aligned}
& I\left(\begin{array}{c}
l_{1} l_{2} \ldots l_{n-1} \\
j_{1} j_{2} \ldots j_{n-1}
\end{array} \mid \xi_{1} \xi_{2}, \ldots, \xi_{n}\right) \\
\equiv & \frac{|A|^{1 / 2}}{(2 \pi)^{n / 2}} \int_{0}^{\infty} \int_{0}^{\infty} \ldots \int_{0}^{\infty} \prod_{i=1}^{n-1}\left(L_{j i}\left(u_{i}, u_{i+1}\right)\right)^{l_{i}} \exp \left\{-\frac{Q}{2}\right\} \prod_{i=1}^{n} d u_{i},
\end{aligned}
$$

where we have put

$$
\begin{aligned}
& L_{j_{i}}\left(\boldsymbol{u}_{i}, \boldsymbol{u}_{i+1}\right)=\boldsymbol{u}_{i}+\boldsymbol{u}_{i+1}, \\
&=\left(\boldsymbol{u}_{i}^{2}+\boldsymbol{u}_{i+1}^{2}\right) /\left(\boldsymbol{u}_{i}+\boldsymbol{u}_{i+1}\right), \\
& Q \equiv \sum_{i=1}^{n} \sum_{j=1}^{n} A_{j_{i}}\left(\boldsymbol{u}_{i}-\xi_{i}\right)\left(\boldsymbol{u}_{j}-\xi_{j}\right) \\
&|A|=\mid A_{i j},
\end{aligned}
$$

when $j_{i}=0$

when $j_{i}=1$

with a positive definite quadratic form $Q$, and non-negative integers $l_{i}(i=$ $0,1,2, \ldots, n)$.

First let us write

$$
Q=\sum_{i=1}^{n} \sum_{j=1}^{n} A_{i j} u_{i} u_{j}-2 \sum_{i=1}^{n} \sum_{j=1}^{n}\left(A_{i j} \xi_{j}\right) u_{i}+\sum_{i=1}^{n} \sum_{j=1}^{n} A_{i j} \xi_{i} \xi_{j} .
$$

Then the change of the order of the integration and the infinite summa- 
tion gives us

$$
\begin{aligned}
& I\left(\begin{array}{c}
l_{1} l_{2} \ldots l_{n-1} \\
j_{1} j_{2} \ldots j_{n-1}
\end{array} \mid \xi_{1} \xi_{2} \ldots \xi_{n}\right) \\
& =\exp \left\{-\frac{1}{2} \sum_{i=1}^{n} \sum_{j=1}^{n} A_{i j} \xi_{i} \xi_{j}\right\} \\
& \cdot \sum_{k_{1}=0}^{\infty} \sum_{k_{2}=0}^{\infty} \ldots \sum_{k n=0}^{\infty} \prod_{i=1}^{n} \frac{\delta_{i}^{k i}}{\Gamma\left(k_{i}+1\right)} \\
& \cdot \frac{A_{\mid}^{1 / 2}}{(2 \pi)^{n / 2}} \int_{0}^{\infty} \int_{0}^{\infty} \ldots \int_{0}^{\infty} u_{h}^{k n} \prod_{i=1}^{n-1} L_{j_{i}}\left(u_{i}, u_{i+1}\right)^{l i} u_{i}^{k i} \exp \left\{-\frac{q}{2}\right\} d u_{1} \ldots d u_{n},
\end{aligned}
$$

where we have put

$$
\begin{aligned}
\delta_{i} & \equiv \sum_{j=1}^{n} A_{i j} \xi_{j} \quad(i=1,2, \ldots, n) \\
q & \equiv \sum_{i=1}^{n} \sum_{j=1}^{n} A_{i j} u_{i} u_{j} .
\end{aligned}
$$

Since $q$ is a positive definite quadratic form, the Jacobi transformation reduces $q$ to its normal form. Indeed let us define

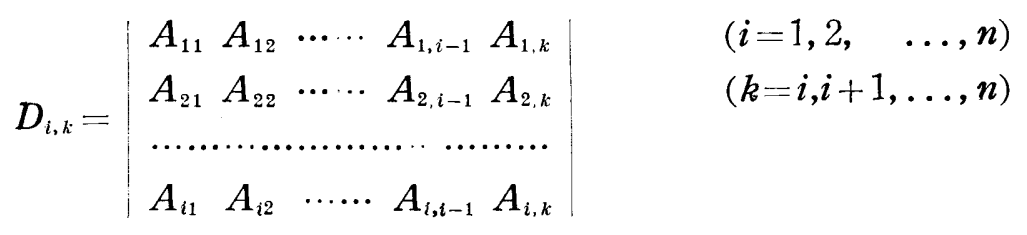

and then let us introce the new system of the variables $\left(z_{1}, z_{2}, \ldots, z_{n}\right)$ such that

$$
z_{i}=\frac{\sqrt{D_{i, i}}}{\sqrt{D_{i-1, i-1}}}\left\{u_{i}+\sum_{k=i+1}^{n} \frac{D_{i, k}}{D_{i, i}} u_{k}\right\} \quad(i=1,2, \ldots, n) .
$$

This yields us

$$
\begin{aligned}
& q \equiv \sum_{i=1}^{n} z_{i} \\
& d u_{1} d u_{2} \ldots d u_{n}=D_{n, n}^{-1 / 2} d z_{1} \ldots d z_{n}=|A|^{-1 / 2} d z_{1} \ldots d z_{n} .
\end{aligned}
$$

The inverse transformation of the transformation $u=\left(u_{1}, u_{2}, \ldots, u_{n}\right)$ into $z=\left(z_{1}, z_{2}, \ldots, z_{n}\right)$ is readily seen to be of the form

$$
\boldsymbol{u}_{i}=\sum_{k=i}^{n} C_{i, k} z_{k} \quad(i=1,2, \ldots, n),
$$

with a certain non-singular triangular matrix $\left(C_{i k}\right)$.

Now the polar coordinate $\left(r, \theta_{1}, \theta_{2}, \ldots, \theta_{n-1}\right)$ is defined by 


$$
z_{1 i}=r\left(\prod_{j=1}^{i-1} \sin \theta^{j}\right) \cos \theta_{i} \equiv r \varphi_{i}(\theta) \quad(i=1,2, \ldots, n-1),
$$

where $0 \leqq \theta_{i}<2 \pi(i=1,2, \ldots, n-2), 0 \leqq \theta_{n-1}<\pi, \theta_{n} \equiv 0$. This yields us

$$
\begin{aligned}
& L_{0}\left(u_{i}, u_{i+1}\right)=r \sum_{j=i}^{n} C_{i, j}^{(0)}\left(\prod_{p=1}^{j-1} \sin \theta_{p}\right) \cos \theta_{j} \equiv r Q_{0}^{(i)}(\theta) \\
& L_{1}\left(u_{i}, u_{i+1}\right) \\
& =r \frac{\left(\sum_{j=1}^{n} C_{i, j}\left(\prod_{p=1}^{j-1} \sin \theta_{p}\right) \cos \theta_{j}\right)^{2}+\left(\sum_{j=i+1}^{n} C_{i+1, j}\left(\prod_{p=1}^{j=1} \sin \theta_{p}\right) \cos \theta_{j}\right)^{2}}{\sum_{k=j}^{n} C_{i, j}^{(0)}\left(\prod_{p=1}^{i-1} \sin \theta_{p}\right) \cos \theta_{j}} \\
& \equiv \boldsymbol{r} \mathfrak{R}_{1}^{(i)}(\theta),
\end{aligned}
$$

where we have put

$$
C_{i, i}^{(0)}=C_{i, i}, \quad C_{i, k}^{(0)}=C_{i, k}+C_{i+1, k} \quad(k \geq i+1) .
$$

We have also

$$
\begin{aligned}
\prod_{i=1}^{n} u_{i}{ }^{k i} & =\boldsymbol{r}^{k_{1}+k_{2}+\cdots+k n} \prod_{i=1}^{n}\left(\sum_{j=\imath}^{n} C_{i j}\left(\prod_{p=1}^{j-1} \sin \theta_{p}\right) \cos \theta_{j}\right)^{k i} \\
& \equiv \boldsymbol{r}^{k_{1}+k_{2}+\cdots+k n} \Re\left(\theta ; k_{1}, k_{2}, \ldots, k_{n}\right) .
\end{aligned}
$$

The use of $(3.11) \sim(3.18)$ in the right-hand side of (3.06) yields us, after integration with respect to $r$ over the domain $0 \leqq r<\infty$, that

$$
\begin{aligned}
& I\left(\begin{array}{llll|llll}
l_{1} & l_{2} & \ldots & l_{n-1} \\
j_{1} & j_{2} & \ldots & j_{n-1}
\end{array} \mid \xi_{1} \xi_{2} \ldots . \quad \xi_{n}\right)
\end{aligned}
$$

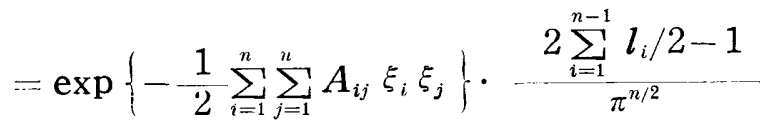

$$
\begin{aligned}
& \text { - } \sum_{k_{1}=0}^{\infty} \sum_{k_{2}=0}^{\infty} \ldots \sum_{k_{n}=0}^{\infty}\left(\prod_{i=1}^{n} \frac{\left(2^{1 / 2} \delta_{i}\right)^{k_{i}}}{\left(\boldsymbol{k}_{i}+1\right)}\right) \Gamma\left(\frac{\sum_{i=1}^{n-1} l_{i}+\sum_{i=1}^{n} \boldsymbol{k}_{i}+n}{2}\right) \\
& \text { - } \frac{1}{(2 \pi)^{n / 2}} \iiint_{D} \prod_{i=1}^{n-1}\left(\mathcal{Q}_{j i}^{(i)}(\theta)\right), k \Re\left(\theta ; k_{1}, k_{2}, \ldots, k_{n}\right) \\
& \text { - } \prod_{j=1}^{n-2} \sin ^{n-1-j} \theta^{j} \prod_{i=1}^{n-1} d \theta_{i},
\end{aligned}
$$

where the domain of integration $\mathfrak{D}$ in $\left(\theta_{1}, \theta_{2}, \ldots, \theta_{n-1}\right)$ is defined as the domain satisfying the follwing inequalities simultaneously:

$$
\sum_{k=i}^{n} C_{i, k}\left(\prod_{j=1}^{k-1} \sin \theta_{j}\right) \cos \theta_{k} \geqq 0 \quad(i=1,2, \ldots, n) .
$$

The integrand in the right-hand side of (3.19) is a rational function of $\sin \theta_{j}$ and $\cos \theta_{j}(j=1,2, \ldots, n-1)$, and therefore can be evaluated by the combination of elementary integrations, although there remain a lot of complexity in their individual cases.

3.2. Let us apply our procedure to the evaluations of the special cases 
of (3.01) when $n=2$. For the convenience let us write

$$
\begin{aligned}
& I\left(\begin{array}{l|l}
1 \\
0
\end{array} \xi_{1}, \xi_{2}\right) \equiv M_{l}\left(\xi_{1}, \xi_{2} ; \sigma_{1}^{2}, \sigma_{2}^{2}, \rho\right) \\
& \equiv \frac{1}{2 \pi \sigma_{1} \sigma_{2}\left(1-\rho^{2}\right)^{1 / 2}} \int_{0}^{\infty} \int_{0}^{\infty}(u+v)^{\imath} \exp \left\{-\frac{Q}{2}\right\} d u d v \\
& I\left(\begin{array}{l|l}
1 \\
0
\end{array} \xi_{1}, \xi_{2}\right) \equiv N_{l}\left(\xi_{1}, \xi_{2} ; \sigma_{1}^{2}, \sigma_{2}^{2}, \rho\right) \\
& \equiv \frac{1}{2 \pi \sigma_{1} \sigma_{2}\left(1-\rho^{2}\right)^{1 / 2}} \int_{0}^{\infty} \int_{0}^{\infty}\left(\frac{u^{2}+v^{2}}{u+v}\right)^{l} \exp \left\{-\frac{Q}{2}\right\} d u d v,
\end{aligned}
$$

where

$$
\begin{aligned}
\frac{Q}{2} & \equiv \frac{1}{2\left(1-\rho^{2}\right)}\left\{\frac{\left(u-\xi_{1}\right)^{2}}{\sigma_{1}^{2}}-2 \rho \frac{\left(u-\xi_{1}\right)\left(v-\xi_{2}\right)}{\sigma_{1} \sigma_{2}}+\frac{\left(v-\xi_{2}\right)^{2}}{\sigma_{2}^{2}}\right\} \\
& =\frac{1}{2\left(1-\rho^{2}\right)}\left(\frac{\xi_{1}^{2}}{\sigma_{1}^{2}}-2 \rho \frac{\xi_{1} \xi_{2}}{\sigma_{1} \sigma_{2}}+\frac{\xi_{2}^{2}}{\sigma_{2}^{2}}\right) \\
& -\frac{u}{\left(1-\rho^{2}\right) \sigma_{1}}\left(\frac{\xi_{1}}{\sigma_{1}}-\rho \frac{\xi_{2}}{\sigma_{2}}\right)-\frac{v}{\left(1-\rho^{2}\right) \sigma_{2}}\left(\frac{\xi_{2}}{\sigma_{2}}-\rho \frac{\xi_{1}}{\sigma_{1}}\right) \\
& +\frac{1}{2\left(1-\rho^{2}\right)}\left(\frac{u^{2}}{\sigma_{1}^{2}}-2 \rho \frac{u v}{\sigma_{1} \sigma_{2}}+\frac{v^{2}}{\sigma_{2}^{2}}\right) .
\end{aligned}
$$

Our method is based upon the expansions to the effect that

$$
\begin{aligned}
& M_{l}\left(\xi_{1}, \xi_{2} ; \sigma_{1}^{2}, \sigma_{2}^{2}, \rho\right) \\
& =\exp \left\{-\frac{1}{2\left(1-\rho^{2}\right)}\left(\frac{\xi_{1}^{2}}{\sigma_{1}^{2}}-2 \rho \frac{\xi_{1} \xi_{2}}{\sigma_{1} \sigma_{2}}+\frac{\xi_{2}^{2}}{\sigma_{2}^{2}}\right)\right\} \\
& \text { - } \sum_{k_{1}=1}^{\infty} \sum_{k_{2}=0}^{\infty} \frac{\left(\frac{\xi_{1}}{\sigma_{1}}-\rho \frac{\xi_{2}}{\sigma_{2}}\right)^{k_{1}}\left(\frac{\xi_{2}}{\sigma_{2}}-\rho \frac{\xi_{1}}{\sigma_{1}}\right)^{k_{2}}}{\Gamma\left(k_{1}+1\right) \Gamma\left(k_{2}+1\right)\left(1-\rho^{2}\right)^{k_{1}+k_{2}}} \\
& \text { - } \frac{1}{2 \pi\left(1-\rho^{2}\right)^{1 / 2}} \int_{0}^{\infty} \int_{0}^{\infty}\left(\sigma_{1} u+\sigma_{2} v\right)^{l} \boldsymbol{u}^{k_{1}} v^{k_{2}} \exp \left\{-\frac{q}{2}\right\} d u d v,
\end{aligned}
$$

and

$$
\begin{aligned}
& N_{l}\left(\xi_{1}, \xi_{2} ; \sigma_{1}^{2}, \sigma_{2}^{2}, \rho\right) \\
& =\exp \left\{-\frac{1}{2\left(1-\rho^{2}\right)}\left(\frac{\xi_{1}^{2}}{\sigma_{1}^{2}}-2 \rho \frac{\xi_{1} \xi_{2}}{\sigma_{1} \sigma_{2}}+\frac{\xi_{2}^{2}}{\sigma_{2}^{2}}\right)\right\} \\
& \cdot \sum_{k_{1}=0}^{\infty} \sum_{k_{2}=0}^{\infty} \frac{\left(\frac{\xi_{1}}{\sigma_{1}}-\rho \frac{\xi_{2}}{\sigma_{2}}\right)^{k_{1}}\left(\frac{\xi_{2}}{\sigma_{2}}-\rho \frac{\xi_{1}}{\sigma_{1}}\right)^{k_{2}}}{\Gamma\left(k_{1}+1\right) \Gamma\left(k_{2}+1\right)\left(1-\rho^{2}\right)^{k_{1}+k_{2}}}
\end{aligned}
$$




$$
\cdot \frac{1}{2 \pi\left(1-\rho^{2}\right)^{1 / 2}} \int_{0}^{\infty} \int_{0}^{\infty}\left(\frac{\sigma^{2} u^{2}+\sigma_{2}^{2} v^{2}}{\sigma_{1} u+\sigma_{2} v}\right)^{l} u^{k_{1}} v^{k_{2}} \exp \left\{-\frac{q}{2}\right\} d u d v
$$

where we have put

$$
\frac{q}{2} \equiv \frac{1}{2\left(1-\rho^{2}\right)}\left(u^{2}-2 \rho u v+v^{2}\right) .
$$

But we have

$$
\begin{aligned}
& \frac{1}{2 \pi\left(1-\rho^{2}\right)^{1 / 2}} \int_{0}^{\infty} \int_{0}^{\infty}\left(\sigma_{1} u+\sigma_{2} v\right)^{l} u^{k_{1}} v^{k_{2}} \exp \left\{-\frac{q}{2}\right\} d u d v \\
& =\sum_{h=0}^{l}{ }_{l} C_{h} \sigma_{1}^{h} \sigma_{2}^{l-h} \\
& \cdot \frac{1}{2 \pi\left(1-\rho^{2}\right)^{1 / 2}} \int_{0}^{\infty} \int_{0}^{\infty} u^{k_{1}+h} v^{k_{2}+l-h} \exp \left\{-\frac{q}{2}\right\} d u d v
\end{aligned}
$$

and

$$
\begin{aligned}
& \frac{1}{2 \pi\left(1-\rho^{2}\right)} \int_{0}^{\infty} \int_{0}^{\infty}\left(\frac{\sigma_{1}^{2} u^{2}+\sigma_{2}^{2} v^{2}}{\sigma_{1} u+\sigma_{2} v}\right)^{l} u^{k_{1}} v^{k_{2}} \exp \left\{-\frac{q}{2}\right\} d u d v \\
& =\sum_{h=0}^{l} C_{h} \sigma_{1}^{2 h} \sigma_{2}^{2(l-h)} \\
& \cdot \frac{1}{2 \pi\left(1-\rho^{2}\right)^{1 / 2}} \int_{0}^{\infty} \int_{0}^{\infty} \frac{u^{k_{1}+2 h} v^{k_{2}+2(l-h)}}{\left(\sigma_{1} u+\sigma_{2} v\right)^{l}} \exp \left\{-\frac{q}{2}\right\} d u d v .
\end{aligned}
$$

In the consequence we have to evaluate the integrals in the right hand sides of (3.27). For this purpose let us make use of the polar coordinate $(r, \theta)$ defined by

$$
r \cos \theta=\frac{1}{\sqrt{2(1+\rho)}}(u+v), \quad r \sin \theta=\frac{1}{v^{\prime} \frac{1}{2(1-\rho)}}(u-v) .
$$

This transformation yields us

$$
\begin{aligned}
& \frac{1}{2 \pi\left(1-\rho^{2}\right)^{1 / 2}} \int_{0}^{\infty} \int_{0}^{\infty} u^{k_{1}+h} v^{k_{2}+l-h} \exp \left\{-\frac{1}{2\left(1-\rho^{2}\right)}\left(u^{2}-2 \rho u v+v^{2}\right)\right\} d u d v
\end{aligned}
$$

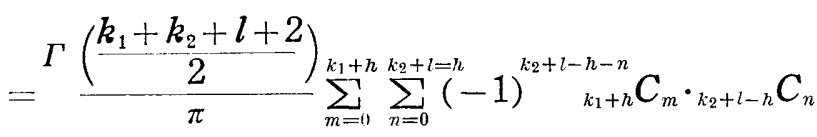

$$
\begin{aligned}
& \text { - }(1+\rho)^{m+n / 2}(1-\rho)^{\left(k_{1}+k_{2}+l-(m+n)\right) / 2} I_{\omega}\left(\boldsymbol{m}+\boldsymbol{n}, \boldsymbol{k}_{1}+\boldsymbol{k}_{2}+\boldsymbol{l}-\boldsymbol{m}-\boldsymbol{n}\right) \text {, }
\end{aligned}
$$

where $I_{\omega}(p, q)$ and $\omega$ means 


$$
\begin{aligned}
& I_{\omega}(p, q) \equiv \int_{0}^{\omega} \cos ^{p} \theta \sin ^{q} \theta d \theta \\
& \omega=\sin ^{-1} \sqrt{\frac{1+\rho}{2}} .
\end{aligned}
$$

Now concerning the right-hand side of (3.28), we should rather prefer to take $\rho$ instead of $\rho$ in view of (1.39) and (1.4.0) and let us make use of the polar coordinate $(r, \theta)$ defined by

$$
r \cos \theta=\frac{1}{\sqrt{2(1+\rho)}}(u-v), r \sin \theta=\frac{1}{\sqrt{2(1-\rho)}}(u+v)
$$

and the auxiliary angle $\varphi_{1}$ defined by

$$
\sin \varphi_{1}=\frac{(2(1+\rho))^{1 / 2}\left(\sigma_{1}-\sigma_{2}\right)}{2\left(\sigma_{1}^{2}+\sigma_{2}^{2}-2 \rho \sigma_{1} \sigma_{2}\right)^{1 / 2}}, 0 \leqq \varphi_{1} \leqq \frac{\pi}{2} .
$$

This device of change of variables gives us now

$$
\begin{aligned}
& \frac{1}{2 \pi\left(1-\rho^{2}\right)^{1 / 2}} \int_{0}^{\infty} \int_{0}^{\infty} \frac{\boldsymbol{u}^{k_{1}+2 h} v^{k_{2}+2(l-h)}}{\left(\sigma_{1} u+\sigma_{2} v\right)^{i}} \exp \left\{-\frac{\boldsymbol{u}^{2}+v^{2}+2 \rho u v}{2\left(1-\rho^{2}\right)}\right\} d u d v \\
& =\frac{\Gamma\left(\frac{k_{1}+k_{2}+l+2}{2}\right)}{2^{l / 2} \pi\left(\sigma_{1}^{2}+\sigma_{2}^{2}-2 \rho \sigma_{1} \sigma_{2}\right)^{l / 2}}
\end{aligned}
$$

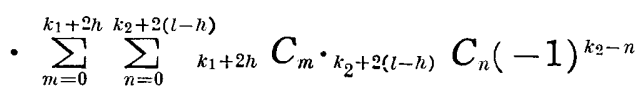

$$
\begin{aligned}
& (1+\rho)^{m+n / 2}(1-\rho)^{\left(k_{1}+k_{2}+2 l-(m+n)\right) / 2} \cdot J_{\omega}\left(\boldsymbol{m}+\boldsymbol{n}, \boldsymbol{k}_{1}+k_{2}+2 l-\boldsymbol{m}-n ; l, \varphi_{1}\right),
\end{aligned}
$$

where $J(p, q ; l, \varphi)$ means

$$
J_{\omega}(p, q ; l, \varphi) \equiv \int_{\omega}^{\pi / 2} \frac{\cos ^{p} \theta \sin ^{q} \theta}{\sin ^{l}(\theta+\varphi)} d \theta .
$$
dures.

The integrals (3.31) and (3.36) can be evaluated by elementary proce-

The special case when $\xi_{1}=\xi_{2}=0$ which corresponds to the null hypothesis that the two populations have the same population means is worth while to be mentioned here.

In view of (3.24) and (3.30) we have

$$
\begin{aligned}
& M_{l}\left(0,0 ; \sigma_{1}^{2}, \sigma_{2}^{2}, \rho\right) \\
& =\frac{\Gamma\left(\frac{l+2}{2}\right)}{\pi} \sum_{h=0}^{l}{ }_{l} C_{h} \sigma_{1}^{h} \sigma_{2}^{l-h} \sum_{m=0}^{h} \sum_{n=0}^{l-h}(-1)^{l-h-n}{ }_{h} C_{m}{ }_{l-h} C_{n}
\end{aligned}
$$




$$
(1+\rho)^{m / 2}(1-\rho)^{(l-(m+n)) / 2} \quad I_{\omega}(m+n, l-(m+n)) .
$$

In particular we have

$$
\begin{aligned}
M_{0}\left(0,0 ; \sigma_{1}^{2}, \sigma_{2}^{2}, \rho\right)= & \frac{1}{\pi} \sin ^{-1} V^{\frac{1+\rho}{2}} \\
M_{1}\left(0,0 ; \sigma_{1}^{2}, \sigma_{2}^{2}, \rho\right)= & \frac{1+\rho}{(2 \pi)^{1 / 2}} \cdot \frac{\sigma_{1}+\sigma_{2}}{2}+\frac{(1-\rho)^{1 / 2}\left(2^{1 / 2}-(1-\rho)^{1 / 2}\right)}{(2 \pi)^{1 / 2}}\left(\sigma_{1}-\sigma_{2}\right) \\
M_{2}\left(0,0 ; \sigma_{1}^{2}, \sigma_{2}^{2}, \rho\right)= & \frac{1}{\pi}\left\{\left(\sigma_{1}+\sigma_{2}\right)^{2}(1+\rho) I_{\omega}(2,0)\right. \\
& +2\left(\sigma_{1}^{2}-\sigma_{2}^{2}\right)\left(1-\rho^{2}\right)^{1 / 2} I_{\omega}(1,1) \\
& \left.+\left(\sigma_{1}-\sigma_{2}\right)^{2}(1-\rho) I_{\omega}(0,2)\right\},
\end{aligned}
$$

where

(3.411) $\quad I_{\omega}(2,0)=\frac{1}{2}\left(\sin ^{-1} \sqrt{\frac{1+\rho}{2}}+\frac{1}{2}\left(1-\rho^{2}\right)^{1 / 2}\right)$

(3.412) $\quad I_{\omega}(1,1)=\frac{1+\rho}{2}$

(3.4.13) $\quad I_{\alpha}(0,2)=\frac{1}{2}\left(\sin ^{-1} \sqrt{\frac{1+\rho}{2}}-\frac{1}{2}\left(1-\rho^{2}\right)^{1 / 2}\right)$.

Similarly, in view of (3.25) and (3.35), we have

$$
\begin{aligned}
& N_{l}\left(0,0 ; \sigma_{1}^{2}, \sigma_{2}^{2},-\rho\right) \\
& =\frac{\Gamma\left(\frac{l+2}{2}\right)}{2^{l / 2} \pi\left(\sigma_{1}^{2}+\sigma_{2}^{2}-2 \rho \sigma_{1} \sigma_{2}\right)^{l / 2} \sum_{h=0}^{l}{ }_{l} C_{h} \sigma_{1}^{2 h} \sigma_{2}^{2(l-h)}} \\
& \cdot \sum_{m=1}^{i n} \sum_{n=0}^{2(l-h)}{ }_{2 h} C_{m}{ }_{2(l-h)} C_{n}(-1)^{n}(1+\rho)^{m / 2}(1-\rho)^{(2 l-(m+n)) / 2} \\
& \cdot J_{\omega}(\boldsymbol{m}, 2 l-(\boldsymbol{m}+\boldsymbol{n}) ; l, \varphi) .
\end{aligned}
$$

In particular we have

$$
\begin{aligned}
& \text { (3.4.3) } \quad N\left(0,0 ; \sigma_{1}^{2}, \sigma_{2}^{2},-\rho\right)=\frac{1}{\pi}\left(\frac{\pi}{2}-\sin ^{-1} \sqrt{\frac{1+\rho}{2}}\right) \\
& \quad N\left(0,0 ; \sigma_{1}^{2}, \sigma_{2}^{2},-\rho\right) \\
& =\frac{1}{2^{3 / 2} \pi^{1 / 2}\left(\sigma_{1}^{2}+\sigma_{2}^{2}-2 \rho \sigma_{1} \sigma_{2}\right)^{1 / 2}}\left[( \sigma _ { 1 } ^ { 2 } + \sigma _ { 2 } ^ { 2 } ) \left\{(1+\rho) J_{\omega}\left(2,0 ; 1, \varphi_{1}\right)\right.\right. \\
& +(1-\rho) J_{\omega}\left(0,2 ; 1, \varphi_{1}\right)
\end{aligned}
$$




$$
\begin{aligned}
& \left.+2\left(\sigma_{1}^{2}-\sigma_{2}^{2}\right)\left(1-\rho^{2}\right)^{1 / 2} J_{\omega}\left(1,1 ; 1, \varphi_{1}\right)\right] \\
& N_{2}\left(0,0 ; \sigma_{1}^{2}, \sigma_{2}^{2},-\rho\right) \\
& =-\frac{(1-\rho)^{2}}{2 \pi\left(\sigma_{1}^{2}+\sigma_{2}^{2}-2 \rho \sigma_{1} \sigma_{2}\right)}\left[\sigma_{1}^{4} \sum_{m=0}^{4}{ }_{4} C_{m}\left(\frac{1+\rho}{1-\rho}\right)^{m / 2} J_{\omega}\left(m, 4-m ; 2, \varphi_{1}\right)\right. \\
& +2 \sigma_{1}^{2} \sigma_{2}^{2} \sum_{m=0}^{2} \sum_{n=0}^{2}{ }_{2} C_{m} \cdot{ }_{2} C_{n}(-1)^{n}\left(\frac{1+\rho}{1-\rho}\right)^{(m+n) / 2} \boldsymbol{J}_{\omega}\left(\boldsymbol{m}+\boldsymbol{n}, 4-\boldsymbol{m}-\boldsymbol{n} ; 2, \varphi_{1}\right) \\
& \left.+\sigma_{2}^{4} \sum_{n=0}^{4}{ }_{4} C_{n}\left(\frac{1+\rho}{1-\rho}\right)^{n / 2}(-1)^{n} J_{\omega}\left(n, 4-n ; 2, \varphi_{1}\right)\right),
\end{aligned}
$$

where $J_{\omega}\left(p, q ; l, \varphi_{1}\right) \quad(p, q=0,1,2,3,4 ; l=1,2,4)$ can be evaluated by elementary calculations.

Specially when $\sigma_{1}^{2}=\sigma_{2}^{2}$, we have

$$
\begin{aligned}
& M_{1}\left(0,0 ; \sigma^{2}, \sigma^{2}, \rho\right)=\frac{(1+\rho) \sigma}{(2 \pi)^{1 / 2}} \\
& N_{1}\left(0,0 ; \sigma^{2}, \sigma^{2},-\rho\right)=\frac{\sigma}{2 \pi^{1 / 2}}\left(\frac{1+\rho}{(1-\rho)^{1 / 2}} \log \frac{\sqrt{2}+\sqrt{1-\rho}}{\sqrt{1+\rho}}-2^{1 / 2} \rho\right) \\
& M_{2}\left(0,0 ; \sigma^{2}, \sigma^{2}, \rho\right)=\frac{2(1+\rho) \sigma^{2}}{\pi}\left(\sin ^{-1} \sqrt{\frac{1+\rho}{2}}+\frac{\left(1-\rho^{2}\right)^{1 / 2}}{2}\right) \\
& N_{2}\left(0,0 ; \sigma^{2}, \sigma^{2}, \rho\right)=\frac{\sigma^{2}}{(1-\rho) \pi}\left(\left(1+\rho+\rho^{2}\right)\left(1-\rho^{2}\right)^{1 / 2}\right. \\
& \quad-\rho(2+\rho)\left(\pi-2 \sin ^{-1} \sqrt{\frac{1+\rho}{2}}\right) .
\end{aligned}
$$

\$4. Numerical evaluations of variances, covariances, eorrelationcoefficients of the multivariate normal distribution associated with a graphical fractile analysis.

Let us consider the special case when the size of a sample is 19. Let $\lambda=0.05 \sim 0.950$ [0.05]. To each of these values of $\lambda$, let us defined $x$ and $z_{i}$ by

$$
\begin{aligned}
& \frac{1}{\sqrt{2 \pi}} \int_{-\infty}^{x i} e^{-t^{2} / 2} d t=\lambda_{i} \equiv 0.05_{i} \\
& \frac{1}{\sqrt{2}} e^{-x^{2} / 2}=z_{i} .
\end{aligned}
$$

The auxiliary table of the $19 \times 19$ matrix $\left(\lambda_{i}\left(1-\lambda_{j}\right) / z_{i} z_{j}\right)(i, j=1,2, \ldots$, 
19) is calculated. Our fractile analysis is here concerned with the following three groupings.

Example 4.1. Let us consider the case $\boldsymbol{n}_{1}^{\prime}=\boldsymbol{n}_{2}^{\prime}=\boldsymbol{n}_{3}^{\prime}=\boldsymbol{n}_{4}^{\prime}=4$ and $\boldsymbol{n}_{5}^{\prime}=3$. For this grouping the modification of (2.27) is involved because the sizes of subgroups are not equal, and we shall define

$$
\begin{aligned}
& \lambda_{k, i}^{(19)}=(4(k-1)+i) / n \\
& \lambda_{k, i}^{(19)}=(16+i) / n
\end{aligned}
$$$$
(k=1 \sim 4 ; i=1 \sim 4)
$$$$
(k=5, i=1,2,3)
$$

and the corresponding $x_{k, i}^{(19)}$ and $z_{k, i}^{(19)}$ according to (4.01) and (4.02) respectively. Let us then calculate

$$
S_{k_{1}, k_{2}}=\sum_{i=1}^{n^{\prime} k_{1}} \sum_{j=1}^{n^{\prime} k_{2}} \frac{\lambda_{k_{1}, i}^{(19)}\left(1-\lambda_{k_{2}}^{(19)}\right)}{\boldsymbol{z}_{k_{1}, i}^{(20)} \boldsymbol{z}_{k_{2}, j}^{(20)}}
$$

for $k_{1}, k_{2}=1,2,3,4$.

According to (2.31), we calculate

$$
\sigma_{\left(k_{1} k_{2}\right)}=\frac{1}{19} \cdot \frac{S_{k_{1}, k_{2}}}{\left(\boldsymbol{n}_{k_{1}}^{\prime} n_{k_{2}}^{\prime}\right)^{1 / 2}}\left(k_{1}, k_{2}=1 \sim 5\right) \text {, }
$$

and hence $\sigma_{(k)}^{2}=\sigma_{(k, k)}$ and $\rho_{\left(k_{1}, k_{2}\right)} \quad(k=1,2,3,4,5)$ according to $(2.321) \sim$ (2.322).

Then we proceed to define $\sigma_{(k)\rangle}^{2}, \sigma_{\left(k_{1}, k_{2}\right)}$ and $\rho_{\left(\left\langle k_{1}, k_{2}\right)\right)}$ for $|\rho|=0.1 \sim 0.9[0.1]$ according to $(2.34) \sim(2.36)$, where we use now the normalised value of $\sigma_{i}^{2}$ $=1$. This example is called as the Case I.

Example 4.2. The Case II treats with the grouping when $\boldsymbol{n}_{1}^{\prime}=4$ and $n_{2}^{\prime}=3 \quad(i=2 \sim 6)$.

Example 4.3. The Case III treats with the grouping when $\boldsymbol{n}_{1}^{\prime}=\boldsymbol{n}_{2}^{\prime}=\boldsymbol{n}_{3}^{\prime}$ $=5$ and $n_{4}^{\prime}=4$.

For each of these two Cases II and III we can calculate $\sigma_{\left(k_{1}, k_{2}\right)}, \rho_{\left(k_{1}, k_{2}\right)}$, $\sigma_{(k)}^{2}, \sigma_{\left\langle\left(k_{1}, k_{2}\right)\right)}^{2}$ and $\rho_{\left(k_{1}, k_{2}\right)}$ similarly as in the Case I.

Table 1, (1) (3) give the values of $\sigma_{\left(k_{1}, k 2\right)}$ and $\rho_{\left(k_{1}, k_{2}\right)}$ for the Cases I, II and III respectively Table 2 gives the values of $\sigma_{\{k\rangle)}$ as functions of $|\rho|$ for these Cases, and Table 3 those of $\rho_{\left(k_{1}, k_{2)}\right)}$. 
Table 1, (1). $\sigma_{\left(k_{1}, k_{2}\right)}$ (upper) and $\rho_{\left(k_{1}, k_{2}\right)}$ (lower) for the Case I.

\begin{tabular}{|c|c|c|c|c|c|}
\hline & $\begin{array}{c}4 \\
(1)\end{array}$ & $\begin{array}{c}4 \\
(2)\end{array}$ & $\begin{array}{c}4 \\
(3)\end{array}$ & $\begin{array}{c}4 \\
(4)\end{array}$ & $\begin{array}{c}3 \\
(5)\end{array}$ \\
\hline $4(1)$ & $\begin{array}{l}0.44921 \\
1.00000\end{array}$ & $\begin{array}{l}0.24381 \\
0.64902\end{array}$ & $\begin{array}{l}0.15284 \\
0.41957\end{array}$ & $\begin{array}{l}0.10503 \\
0.27318\end{array}$ & $\begin{array}{l}0.06223 \\
0.14813\end{array}$ \\
\hline $4(2)$ & & $\begin{array}{l}0.31416 \\
1.00000\end{array}$ & $\begin{array}{l}0.22998 \\
0.75493\end{array}$ & $\begin{array}{l}0.15803 \\
0.49154\end{array}$ & $\begin{array}{l}0.09364 \\
0.26654\end{array}$ \\
\hline $4(3)$ & & & $\begin{array}{l}0.29540 \\
1.00000\end{array}$ & $\begin{array}{l}0.23218 \\
0.74473\end{array}$ & $\begin{array}{l}0.13757 \\
0.40383\end{array}$ \\
\hline $4(4)$ & & & & $\begin{array}{l}0.32903 \\
1.00000\end{array}$ & $\begin{array}{l}0.23181 \\
0.64474\end{array}$ \\
\hline $3(5)$ & & & & & $\begin{array}{l}0.39289 \\
1.00000\end{array}$ \\
\hline
\end{tabular}

Table 1, (2). $\sigma_{\left(k_{1}, k_{2}\right)}$ (upper) and $\rho_{\left(k_{1}, k_{2}\right)}$ (lower) for the Case II.

\begin{tabular}{c|c|c|c|c|c|c}
\hline & 4 & 3 & 3 & 3 & 3 & 3 \\
& $(1)$ & $(2)$ & $(3)$ & $(4)$ & $(5)$ & $(6)$ \\
\hline $3(1)$ & 0.44921 & 0.22461 & 0.15379 & 0.11425 & 0.08664 & 0.06223 \\
& 1.00000 & 0.67121 & 0.47897 & 0.35310 & 0.25156 & 0.14813 \\
$3(2)$ & & 0.24929 & 0.19099 & 014189 & 0.10760 & 0.07729 \\
& & 1.00000 & 0.79849 & 0.58865 & 0.41938 & 0.24695 \\
$3(3)$ & & & 0.22949 & 018734 & 0.14207 & 0.10204 \\
& & & 1.00000 & 0.81004 & 0.57711 & 0.33983 \\
$3(4)$ & & & & 0.23306 & 0.19482 & 0.13993 \\
& & & & 1.00000 & 0.78530 & 0.46243 \\
$3(5)$ & & & & & 0.26406 & 0.21540 \\
& & & & & 1.00000 & 0.66874 \\
$3(6)$ & & & & & & 0.39289 \\
& & & & & & 1.00000 \\
\hline
\end{tabular}

Table 1, (3). $\sigma_{\left(k_{1}, k_{2}\right)}$ (upper) and $\rho_{\left(k_{1}, k_{2}\right)}$ (Iower) for the Case III.

\begin{tabular}{c|c|c|c|c}
\hline & 5 & 5 & 5 & 4 \\
& $(1)$ & $(2)$ & $(3)$ & $(4)$ \\
\hline $5(1)$ & 0.49877 & 0.26818 & 0.16052 & 0.09082 \\
& 1.00000 & 0.62854 & 0.41564 & 0.19187 \\
$5(2)$ & & 0.36500 & 0.26259 & 0.14858 \\
& & 1.00000 & 0.79484 & 0.36692 \\
$5(3)$ & & & 0.29902 & 0.25752 \\
& & & 1.00000 & 0.70265 \\
$4(4)$ & & & & 0.44921 \\
& & & & 1.00000 \\
\hline
\end{tabular}


Table 2, (1). $\sigma_{((k))}$ for the Case I.

\begin{tabular}{c|c|c|c|c|c}
\hline$|\rho|$ & $\begin{array}{c}4 \\
\sigma_{((1))}\end{array}$ & $\begin{array}{c}4 \\
\sigma((2))\end{array}$ & $\begin{array}{c}4 \\
\sigma_{((3))}\end{array}$ & $\begin{array}{c}4 \\
\sigma_{((4))}\end{array}$ & $\begin{array}{c}3 \\
\sigma_{((5))}\end{array}$ \\
\hline 0.1 & 0.50199 & 0.50064 & 0.50045 & 0.50079 & 0.57787 \\
0.2 & 0.50791 & 0.50256 & 0.50181 & 0.50315 & 0.57941 \\
0.3 & 0.51762 & 0.50574 & 0.50407 & 0.50706 & 0.58197 \\
0.4 & 0.53092 & 0.51016 & 0.50721 & 0.51249 & 0.58554 \\
0.5 & 0.54754 & 0.51579 & 0.51122 & 0.51938 & 0.59010 \\
0.6 & 0.56720 & 0.52259 & 0.51608 & 0.52769 & 0.59563 \\
0.7 & 0.58959 & 0.53051 & 0.52177 & 0.53733 & 0.60209 \\
0.8 & 0.61441 & 0.53950 & 0.52826 & 0.54825 & 0.60947 \\
0.9 & 0.64137 & 0.54951 & 0,53551 & 0.56037 & 0.61772 \\
\hline
\end{tabular}

Table 2, (2). $\sigma_{((k))}$ for the Case II.

\begin{tabular}{c|c|c|c|c|c|c}
\hline$|\rho|$ & $\begin{array}{c}4 \\
\sigma_{((1))}\end{array}$ & $\begin{array}{c}3 \\
\sigma_{((2))}\end{array}$ & $\begin{array}{c}3 \\
\sigma_{((3))}\end{array}$ & $\begin{array}{c}3 \\
\sigma_{((4))}\end{array}$ & $\begin{array}{c}3 \\
\sigma_{((5))}\end{array}$ & $\begin{array}{c}3 \\
\sigma_{((6))}\end{array}$ \\
\hline 0.1 & 0.50199 & 0.57662 & 0.57645 & 0.57648 & 0.57675 & 0.57787 \\
0.2 & 0.50791 & 0.57443 & 0.57374 & 0.57387 & 0.57495 & 0.57941 \\
0.3 & 0.51762 & 0.57076 & 0.56920 & 0.56948 & 0.57193 & 0.58197 \\
0.4 & 0.53092 & 056558 & 0.56278 & 0.56328 & 0.56767 & 0.58554 \\
0.5 & 0.54754 & 0.55886 & 0.55441 & 0.55522 & 0.56215 & 0.59010 \\
0.6 & 0.56720 & 0.55052 & 0.54401 & 0.54519 & 0.55533 & 0.59563 \\
0.7 & 0.58959 & 0.54051 & 0.53146 & 0.53310 & 0.54717 & 0.60209 \\
0.8 & 0.61441 & 0.52872 & 0.51660 & 0.51881 & 0.53759 & 0.60947 \\
0.9 & 0.64137 & 0.51503 & 0.49922 & 0.50211 & 0.52652 & 0.61772 \\
\hline
\end{tabular}

Table 2, (3). $\sigma_{((k))}$ for the Case III.

\begin{tabular}{c|c|c|c|c}
\hline $\mid \rho_{i}$ & $\begin{array}{c}5 \\
\sigma_{((1))}\end{array}$ & $\begin{array}{c}5 \\
\sigma_{((2))}\end{array}$ & $\begin{array}{c}5 \\
\sigma_{((3))}\end{array}$ & $\begin{array}{c}4 \\
\sigma_{((1))}\end{array}$ \\
\hline 0.1 & 0.20299 & 0.20165 & 0.20099 & 0.25199 \\
0.2 & 0.21195 & 0.20660 & 0.20396 & 0.25797 \\
0.3 & 0.22689 & 0.21485 & 0.20891 & 0.26793 \\
0.4 & 0.24780 & 0.22640 & 0.21584 & 0.28187 \\
0.5 & 0.27469 & 0.24125 & 0.22476 & 0.29980 \\
0.6 & 0.30756 & 0.25940 & 0.23565 & 0.32172 \\
0.7 & 0.34640 & 0.28085 & 0.24852 & 0.34761 \\
0.8 & 0.39122 & 0.30560 & 0.26338 & 0.37749 \\
0.9 & 0.44201 & 0.33365 & 0.28021 & 0.41136 \\
\hline
\end{tabular}


Table 3. $\rho((i, j))=\frac{\sigma((i, j))}{\sigma((i)) \sigma_{((j))}} \quad(i \neq j)$ for the Cases I, II and III.

\begin{tabular}{|c|c|c|c|c|c|c|}
\hline \multirow{2}{*}{$|\rho|$} & \multicolumn{3}{|c|}{$(1,2)$} & \multicolumn{3}{|c|}{$(1,3)$} \\
\hline & I & II & III & I & II & III \\
\hline $\begin{array}{l}0.1 \\
0.2 \\
0.3 \\
0.4 \\
0.5 \\
0.6 \\
0.7 \\
0.8 \\
0.9\end{array}$ & $\begin{array}{l}0.00970 \\
0.03821 \\
0.08382 \\
0.14403 \\
0.21583 \\
0.29612 \\
0.38196 \\
0.47075 \\
0.56034\end{array}$ & $\begin{array}{l}0.00776 \\
0.03079 \\
0.06842 \\
0.11968 \\
0.18351 \\
0.25895 \\
0.34536 \\
0.44252 \\
0.55077\end{array}$ & $\begin{array}{l}0.01326 \\
0.05126 \\
0.10932 \\
0.18116 \\
0.26044 \\
0.34181 \\
0.42131 \\
0.49639 \\
0.56566\end{array}$ & $\begin{array}{l}0.00608 \\
0.02399 \\
0.05272 \\
0.09081 \\
0.13650 \\
0.18797 \\
0.24345 \\
0.30138 \\
0.36045\end{array}$ & $\begin{array}{l}0.00531 \\
0.02111 \\
0.04698 \\
0.08235 \\
0.12665 \\
0.17942 \\
0.24049 \\
0.31009 \\
0.38904\end{array}$ & $\begin{array}{l}0.00795 \\
0.03088 \\
0.06635 \\
0.11105 \\
0.16150 \\
0.21465 \\
0.26807 \\
0.32004 \\
0.36944\end{array}$ \\
\hline \multirow{2}{*}{$\rho$} & \multicolumn{3}{|c|}{$(1,4)$} & \multicolumn{3}{|c|}{$(1,5)$} \\
\hline & I & II & III & I & II & III \\
\hline $\begin{array}{l}0.1 \\
0.2 \\
0.3 \\
0.4 \\
0.5 \\
0.6 \\
0.7 \\
0.8 \\
0.9\end{array}$ & $\begin{array}{l}0.00418 \\
0.01644 \\
0.03601 \\
0.06176 \\
0.09233 \\
0.12633 \\
0.16244 \\
0.19955 \\
0.23670\end{array}$ & $\begin{array}{l}0.00395 \\
0.01568 \\
0.03488 \\
0.06113 \\
0.09395 \\
0.13301 \\
0.17811 \\
0.22939 \\
0.28736\end{array}$ & $\begin{array}{l}0.00402 \\
0.01554 \\
0.03315 \\
0.05498 \\
0.07912 \\
0.10394 \\
0.12825 \\
0.15125 \\
0.17252\end{array}$ & $\begin{array}{l}0.00215 \\
0.00846 \\
0.01859 \\
0.03203 \\
0.04815 \\
0.06631 \\
0.08590 \\
0.10636 \\
0.12723\end{array}$ & $\begin{array}{l}0.00299 \\
0.01187 \\
0.02634 \\
0.04600 \\
0.07037 \\
0.09902 \\
0.13160 \\
0.16788 \\
0.20782\end{array}$ & $\begin{array}{l}- \\
- \\
- \\
- \\
- \\
-\end{array}$ \\
\hline \multirow{2}{*}{$|\rho|$} & \multicolumn{3}{|c|}{$(1,6)$} & \multicolumn{3}{|c|}{$(2,3)$} \\
\hline & I & II & III & I & II & $11 I$ \\
\hline $\begin{array}{l}0.1 \\
0.2 \\
0.3 \\
0.4 \\
0.5 \\
0.6 \\
0.7 \\
0.8 \\
0.9\end{array}$ & $\begin{array}{l}- \\
- \\
- \\
- \\
- \\
-\end{array}$ & $\begin{array}{l}0.00215 \\
0.00846 \\
0.01859 \\
0.03203 \\
0.04815 \\
0.06631 \\
0.08590 \\
0.10636 \\
0.12723\end{array}$ & $\begin{array}{l}- \\
- \\
- \\
- \\
- \\
-\end{array}$ & $\begin{array}{l}0.00918 \\
0.03648 \\
0.08119 \\
0.14220 \\
0.21804 \\
0.30698 \\
0.40711 \\
0.51645 \\
0.63303\end{array}$ & $\begin{array}{l}0.00575 \\
0.02318 \\
0.05291 \\
0.09600 \\
0.15410 \\
0.22957 \\
032578 \\
0.44751 \\
0.60167\end{array}$ & $\begin{array}{l}0.01304 \\
0.05117 \\
0.11155 \\
0.19006 \\
0.28192 \\
0.38235 \\
0.48703 \\
0.59237 \\
0.69563\end{array}$ \\
\hline \multirow{2}{*}{$|\rho|$} & \multicolumn{3}{|c|}{$(2,4)$} & \multicolumn{3}{|c|}{$(2,5)$} \\
\hline & I & II & III & I & II & III \\
\hline $\begin{array}{l}0.1 \\
0.2 \\
0.3 \\
0.4 \\
0.5 \\
0.6 \\
0.7 \\
0.8 \\
0.9\end{array}$ & $\begin{array}{l}0.00630 \\
0.02500 \\
0.05546 \\
0.09671 \\
0.14748 \\
0.20631 \\
0.27165 \\
0.34195 \\
0.41570\end{array}$ & $\begin{array}{l}0.00427 \\
0.01722 \\
0.03929 \\
0.07126 \\
0.11432 \\
0.17018 \\
0.24128 \\
0.33105 \\
0.44442\end{array}$ & $\begin{array}{l}0.00659 \\
0.02574 \\
0.05573 \\
0.09410 \\
0.13811 \\
0.18515 \\
0.23300 \\
0.27996 \\
0.32484\end{array}$ & $\begin{array}{l}0.00324 \\
0.01286 \\
0.02863 \\
0.05016 \\
0.07691 \\
0.10830 \\
0.14365 \\
0.18227 \\
0.22345\end{array}$ & $\begin{array}{l}0.00324 \\
0.01303 \\
0.02967 \\
0.05362 \\
0.08562 \\
0.12670 \\
0.17827 \\
0.24228 \\
0.32140\end{array}$ & $\begin{array}{l}- \\
- \\
- \\
- \\
- \\
-\end{array}$ \\
\hline
\end{tabular}


Table 3. $\rho_{((i, 2))}=\frac{\sigma_{((i, i))}}{\sigma((i)) \sigma_{((j))}} \quad(i \neq j)$ for the Cases I, II and III.

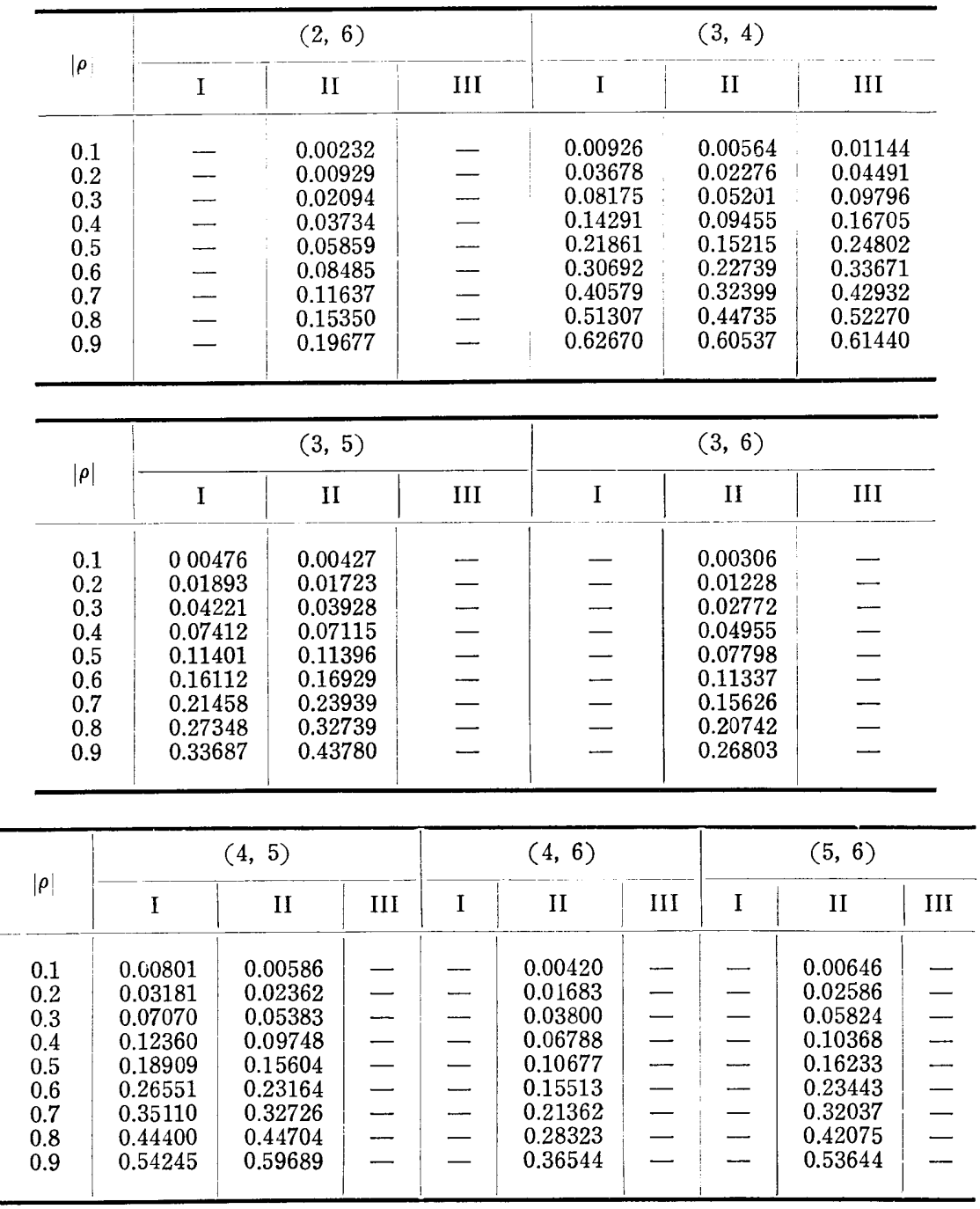

\section{Literatures}

MAHALA NOBIs, P. C.: [1] A method of fractile graphic analysis with some surmises of results, Lectures in Japan: Fractile Graphic Analysis, Indian Statistical Institute, November 1958, JFG 1 JFG 6.

[2] National sample survey: 7th and 9th Rounds, All-India Rural, Lectures in Japan: Consumption, Indian Statistical Institue, November 1958, JC1-IC7.

Mosteller, F.: [1] On some 'inefficient' statistics, Ann. Math. Stat., 17 (1941), 377-408.

Takeuchi, K.: [1] On the lecture by Prof. Mahalanobis, Sugaku no Ayumi, 6 (1958), 196-201 (in Japanese). 